\title{
Étude synoptique des différents types d'éoliennes
}

\author{
L. VADO'T \\ INGENTEUR-CONSEIL ACX ËTABLISSEMENTS NEYHPC
}

English text, p. 204

\begin{abstract}
Après aboir passé en revue toutes les possibilités d'utiliser l'énergie du vent pour l'entrainement de machines tournantes, l'auteur effectue la critique des différentes solutions du point de vne de leur rendement et de lenxs caractéristiques.

II résulte de cet examen que la machine du type helice, a axe parallele da la direction diu vent, est la seule industriellement intéressante. Les differents perfectionnements apportes à ce lupe d'appareil sont présentés. Tontes les solnlions balables ponr la réalisation des princi-
\end{abstract}

\begin{abstract}
paux organes sonf données sous forme de tableau (pylone, roue, réglage, orientation, transmission d'énergie).

Un paragraphe est ensuite consacré à chacune des réalisations possibles qui paraissent intéressantes, tant du point de vue de l'économie de la construction que de la facilité d'exploitation: moulin hollandais amélioré, machine i voiles, machine à pales crenses, machines $\dot{a}$ pales fixes, machine dite « $\dot{a}$ rone de bicycletle ", machine it pales orientables, abec liatson élastique on abec servo-motent.
\end{abstract}

Lorsqu'on se trouve au débul de l'évolution d'une technique, le manque d'expérience ne permettant pas de faire les éliminations nécessaires, un grand nombre de lypes de machines possibles se présentent au chercheur.

Si dans le domaine des éoliennes de petite puissance l'expérience a déjà permis de mettre en avant certaines dispositions et de s'orienter. ver's l'oplimum économique, il n'en est pas de même pour les éoliennes de grande puissance. Dans ce cas, en effet, le nombre des réalisations est encore trop faible pour que puissent se dégager des indications suffisamment nettes sur la voie à suivre.

Il serait donc opportun d'examiner l'ensemble des solutions possibles et d'en effectuer la critique. Ce travail doit permettre d'éviter d'inutiles retours en arrière et indiquer la voie dans laquelle les études ont le plus de chance d'abouLir.

Une classification ordonnce des différents schémas de transformation de l'énergie du vent en énergie utilisable fera ressortir les principaux dispositifs mécaniques dont les caractéristiques et les particularités seront ensuite examinées. Chaque problème de détail comportant aussi plusieurs solutions, il sera nécessaire de les passer en revue et d'en faire la critique. Au terme de cette étude on verra apparaître un certain nombre de machines que des raisons techniques et économiques permettent de considérer comme dignes d'intérêt. Il serait certes prématuré de désigner d'emblée la solution la plus favorable, pour autant d'ailleurs qu'il n'y en ait qu'une, mais il est probable qu'une telle solution appartiendra au groupe des machines qui se dégagera de cet examen. 


\section{CLASSIFICATION DES DIFFÉRENTS TYPES D'ÉOLIENNES}

Nous n'envisagerons ici que les machines destinées à transformer l'énergie cinétique du vent en énergie mécanique utilisable sur l'arbre d'une machine réceptrice.

Dans tous les cas, l'énergie est prélevée sur le vent au moyen de pales ou d'aubages appropriés qui se déplacent dans une certaine direction. On peut classer les éoliennes en deux catégories d'après la direction de déplacement de l'aubage par rapport à la direction du vent.

$1^{\circ}$ Machines dans lesquelles les aubes motrices se déplacent suivant la direction du vent;

$2^{\circ}$ Machines dans lesquelles les aubes motrices se déplacent perpendiculairement à la direction du vent (fig. 1).

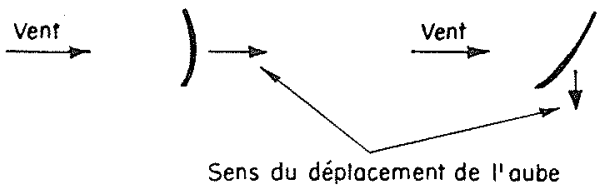

FIG. 1

\section{Machines du premier type :}

\section{Aubes motrices se déplaçant suivant la direction du vent.}

$\mathrm{Si}$ on appelle $v$ la vitesse du vent et $l$ la vitesse de déplacement de l'aubage moteur, ces machines sont caractérisces par la relation :

$$
u<v
$$

On montre par le calcul que le rendement de ces machines est maximum lorsque $u=(v / 3)$; il s'agit donc d'ćoliennes très lentes. L'axe de la roue motrice est perpendiculaire à la direction du vent, une partie seulement de l'aubage est motrice, l'autre au contraire devant aller à contre-courant, il est nécessaire de prévoir un dispositif pour réduire l'effet de freinage qui en résulte. C'est ce dispositif qui caractérise les différentes machines. Nous distinguerons:

a) Les machines à simple traînée, dans lesquelles l'aubage allant en sens contraire du vent est soustrait à l'action de celui-ci.

b) Les machines à différence de traînée, dans lesquelles la forme de l'aubage est telle que sa traînée est plus faible contre le vent que dans le sens du vent.

\section{a) Machines a simples trainées.}

1. Machine à écran : Un écran convenablement placé élimine l'action du vent sur les aubages allant à contre-courant (fig. 2).
2. Machine ḋ clapets battant: les aubages sont articulés sur des axes parallèles à l'axe de la roue. Une cheville d'arrêt disposée près de chaque aubage, retient celui-ci lorsqu'il sup-

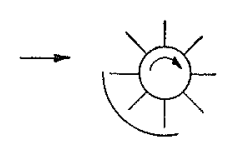

FIG. 2

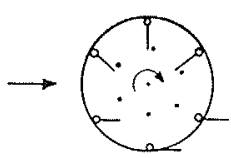

FIG. 3

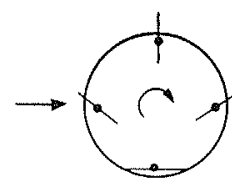

FIG. 4 porte la poussée motrice, mais le laisse libre de se mettre en drapeau dans le vent pour la course de retour (fig. 3 ).

3. Machine à variation cyclique d'orientation des aubes: par un mécanisme épicycloïdal l'aube tourne autour de son axe à demi-vitesse de la roue. On obtient ainsi un effet analogue à celui de la machine à clapet battant, mais sans choc (fig. 4).

\section{b) Machines a différence de trrainée.}

4. Le moulinet à coupelles est le type parfait de ce genre de machines. La forme et le nombre des aubages peuvent évidemment varier d'un type à l'autre (fig. 5).

5. Machine à flux transversal: Ces machines constituent un type de transition entre les classes I et II. Une partie de l'aubage fonctionne grâce à la différence de traînée, mais,

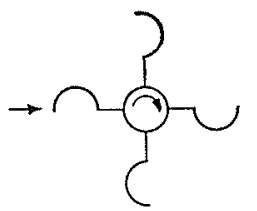

FiG. 5

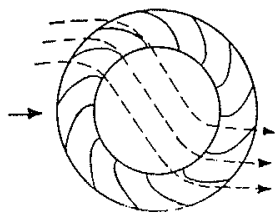

Fig. 6

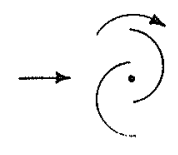

Fic. 7 d'autre part, un flux d'air traverse la machine et travaille deux fois dans l'aubage suivant le schéma analogue à celui des turbines hydrauliques Banki (fig. 6).

6. Rotor de Savonius : Ce genre de machme, qui dérive du moulinet à coupelles, présente une parenté avec la machine à flux transversal et constitue un type de transition très net puisque la vitesse périphérique peut arriver à dépasser la vitesse du vent dans certains cas (fig. 7). 


\section{Machines du deuxième type :}

\section{Aubes motrices se déplaçant perpendiculairement au vent.}

Pour ce type de machines la vitesse $u$ de déplacement de l'aubage moteur peut ètre plus grande que la vitesse du vent. La poussée motrice sur l'aubage qui, dans la catégorie précédente, était une simple traînée de forme, s'obtient ici par déviation de la veine d'air et s'accompagne d'une circulation des vitesses autour du profil de l'aubage. Cette circulation génératrice de poussée motrice s'obtient le plus généralement par la combinaison de l'incidence du courant relatif et de la forme du profil, mais elle peut également ètre obtenue par l'emploi d'un dispositif comme le rotor de Flettner.

Les machines de celte classe se différencient par le mode de déplacement de l'aubage moteur et par les combinaisons mécaniques que ce déplacement nécessite.

\section{a) Machines a Translation.}

7. Machine dite «tapis roulant»: Dans cette machine, une grille d'aubes montée sur un système de courroies passées sur deux poulies se déplace perpendiculairement au vent. Un mécanisme de renversement des aubes permet d'obtenir une poussée motrice sur les deux brins. Le vent traverse donc deux fois le dispositif (fig. 8).

8. Machine d̀ profil oscillant: Une aile dont l'axe est perpendiculaire au vent est montée sur un ensemble de bielles et manivelles dé-

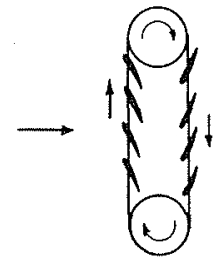

FIG. 8

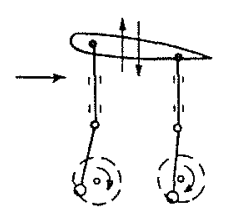

FiG, 9

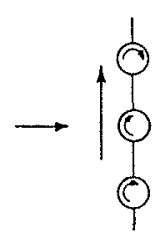

Frg. 10 calées qui lui donne un mouvement oscillant avec poussée dirigée tantôt dans un sens tantôt dans l'autre (fig. 9).

9. Machine a train de rotors: Des rotors de Flettner perpendiculaires au vent peuvent se déplacer sur des charriots auxquels ils transmettent la force motrice. (Dispositif de Madaras) (fig. 10).

b) Machines a variation Cyclioue D'inCIDENCE.

Ces machines sont également appelées panémones. Il ne faut pas confondre avec des ma- chines d'aspect analogue fonctionnant par trâ̂née. Du point de vue aérodynamique, elles présentent une grande parenté avec la machine à tapis roulant. Le vent doit traverser deux fois le système moteur. Cette division par deux de la chute motrice entraine une mauvaise utilisation de l'aubage.

10. Panémone à variation cylique d'orientation; Dans ces machines, l'aubage est moteur uniquement sur les ares $A B$ et $C D$. Le vent traverse deux fois l'aubage. La variation d'incidence sur les profils est obtenue ici par une rotation convenable des aubes (fig. 11).

11. Panémone à aubes fixes: Ces machines ne sont réalisables que pour une vitesse $u$ beaucoup plus grande que la vitesse du vent. La variation d'incidence est due à la combinaison des vitesses, l'incidence ne garde une valeur acceptable que si la vitesse du rotor est assez grande; aussi est-il nécessaire de lancer la machine pour « l'accrocher», ou de

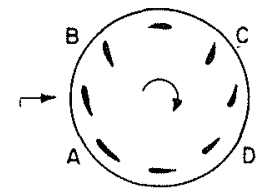

Frg. 11

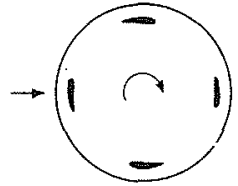

FIG. 12

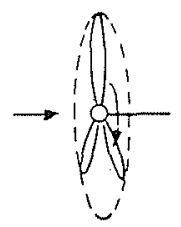

Fig. 13 prévoir un dispositif domnant un couple suffisant au démarrage. On peut, avec des profils convenables et par la disposition des aubes suivant les génératrices d'un cône, assurer un démarrage en utilisant la différence de traînée des profils (fig. 12).

\section{c) MaChines a AXE PARALlìle AU VENT.}

12. Dans ces machines, l'aubage tournant autour d'un axe parallèle au vent possède toujours la même orientation par rapport au vent; le fonctionnement est donc parfaitement continu. La forme des aubes peut varier dans de grandes proportions, elles peuvent être constituées par des rotors de Flettner. La vitesse de déplacement des aubes peut être beaucoup plus grande que la vitesse du vent (fig. 13).

\section{Valeurs des différents types d'éoliennes}

La qualité essentielle exigée d'une éolienne est la possibilité de produire le maximum d'énergie possible, pour un vent donné, avec une construction aussi économique que possible. Pour 
porter un jugement sur la valeur des différentes machines, nous examinerons les points suivants :

1. Vitesse: La vitesse sera caractérisée par le rapport de la vitesse périphérique $u$ (ou de translation) de l'aubage à la vitesse $v$ du vent. Plus ce rapport sera grand, plus la machine tournera vite et plus le prix des appareils entraìnés (multiplicateur, génératrice, etc.) sera laible.

$2^{\circ}$ Coefficient d'utilisation de l'aubage : Ce coefficient est le rapport de la section $\mathrm{S}$ de la veine d'air motrice à la surface $\mathrm{S}_{a}$ développée de l'cnsemble de l'aubage.

Il est évident que plus ce rapport sera grand, plus la machine sera économique. Dans le cas des machines à axe parallèle au vent, la surface $S$ correspond à l'aire balayée par des pales : par contre, dans les machines à trainée, cette surface est nettement plus petite que la surface balayée, puisque l'aube n'est motrice que sur une fraction de tour seulement.

$3^{\circ}$ Caractéristiques mécaniques : Simplicité de construction, nécessité de l'orientation dans le vent, etc., sont autant de facteurs intervenant: pour modifier le prix de la machine.

Etant donné le caractère de rusticité que doit posséder une éoliemne pour fonctionner dans de bonnes conditions sans entretien particulier, il faut attacher une grande importance à la simplicité du mécanisme.

Il y aurait lieu également de faire intervenir le rendement aérodynamique de la machine. Ce rendement, facile à évaluer pour les machines a axe parallèle au vent, est d'une définition trìs difficile pour les machines de la première catégorie; aussi nous paraîtil plus opportun de faire intervenir le coefficient d'utilisation de l'aubage, étant donné le caractère très général de l'examen envisagé.

Les caractéristiques des types de machines classés précédemment sont consignées dans le tableau de la page 193 ci-contre.

Ce tableau (page 193), basé uniquement sur le fonctionnement normal, sans tenir compte du comportement par grand vent, permet d'estimer la valeur des différentes solutions.

Dans la première catégorie (machines dont l'aubage moteur se déplace parallèlement au vent) on ne trouve que des machines extrêmement lentes et d'un très mauvais coefficient d'utilisation de l'aubage. Toutes ces machines doivent être rejetées. Seul le retor de Savonius, par sa simplicité et l'absence de mécanisme d'orientation, peut trouver des applications de très petite puissance (aérateur de wagon, par exemple).
Dans la deuxième catégorie, on trouve des machines plus rapides et d'un meilleur coefficient d'utilisation. La machine à tapis roulant nécessite un mécanime délicat, une charpente coûteuse difficile à orienter. Cette machine, comme toutes les éoliennes à double passage du vent à travers l'aubage est d'un assez mauvais rendement; sa vitesse ne peut être très élevée à cause du double passage.

La machine à profil oscillant est également compliquée et difficile à orienter. La machine à trains de rotor n'est qu'une curiosité. Il sera en effet presque toujours plus avantageux d'utiliser des profils d'ailes que des rotors de Flettner.

Les panémones à variation cyclique d'orientation nécessitent un mécanisme compliqué et ont par ailleurs les défauts des machines à double passage. Les panémones à aubes fixes constituent un perfectionnement par la simplicité de construction et labsence de dispositif d'orientation, mais la vitesse, le coefficient d'utilisation et le rendement sont nettement plus faibles que pour la solution suivante. En outre, le lancement de l'appareil est à peu près nécessaire. La simplicité de cet appareil disparaît en partie si on doit entraîner une machine à vitesse constante et si on considère le comportement par grand vent.

Reste la solution de la machine à axe parallèle an vent (machine du type hélice). La vitesse atteinte et le coefficient d'utilisation de l'aubage sont tels que cette machine surclasse complètement tontes les autres. Elle se prête en outre, comme on le verra, à de nombreuses variantes pour le réglage de la puissance et de la vitesse, ce que les autres machines ne font pas, ou mal, et ce qui est absolument nécessaire pour les éoliennes d'une certaine importance. Enfin elle est pratiquement la seule à permettre par des mécanismes simples une tenue convenable aux très grands rents.

Cel examen rapide montre qu'il n'existe pas dans l'état actuel de la technique, de machines capables de concurrencer efficacement les machines genre hélice.

\section{Modifications apportées aux machines hélices.}

Avant d'approfondir la constitution des machines hélices, nous allons examiner rapidement quelques dispositions imaginées pour perfectionner ces machines. Les principaux soucis des inventeurs dans ce domaine sont l'accroissement de la vitesse de rotation de la machine réceptrice. 


\begin{tabular}{|c|c|c|c|c|}
\hline TYPE DE MACHINE & $u / 0$ & $\mathrm{~S} / \mathrm{S}_{a}$ & $\begin{array}{l}\text { NÉCESSTTE } \\
\text { ORIENTATION }\end{array}$ & OBSERVATIONS \\
\hline 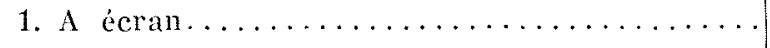 & 0,2 à 0,6 & 0,2 à 0,3 & oui & simple \\
\hline 2. A clapets battants.... & $0,2 \dot{a} 0,6$ & 0,2 & oui & $\begin{array}{l}\text { chocs } \\
\text { possibles. }\end{array}$ \\
\hline 3. A variation cyclique d'orientation....... & 0,2 à 0,6 & 0,2 & oui & $\begin{array}{l}\text { mécanisme } \\
\text { compliqué }\end{array}$ \\
\hline 4. A différence de traince....... & 0,3 à 0,6 & 0,2 & non & simple \\
\hline 5. A flux transversal........... & $0,3 \dot{a} \quad 0,4$ & 0,1 & non & simple \\
\hline 6. Savonius ....... & 0,5 à 1,5 & 0,5 à 0,8 & non & simple \\
\hline 7. Tapis roulant... & 2 à 3 & 1 à 2 & oui & $\begin{array}{l}\text { mécanisme } \\
\text { compliqué }\end{array}$ \\
\hline 8. Profil oscillant... & 0,5 & 0,5 & oui & $\begin{array}{l}\text { mécanisme } \\
\text { compliqué }\end{array}$ \\
\hline 9. Train de rotors... & 2 & $2 \dot{a} 3$ & oui & $\begin{array}{l}\text { nécessite l'entraî- } \\
\text { nement des rotors. } \\
\text { Curiosité. }\end{array}$ \\
\hline 10. Panémone à variation cyclique d'orientation. & $2 \dot{a} 3$ & $1 \dot{a} 2$ & oui & $\begin{array}{l}\text { mécanisme } \\
\text { compliqué }\end{array}$ \\
\hline 11. Panémone à aubes fixes. & $3 \dot{a} 4$ & $2 \dot{a} 3$ & non & $\begin{array}{l}\text { simple, nécessité de } \\
\text { lancement. }\end{array}$ \\
\hline 12. Axe parallèle au vent, machine hélice. & $1 \grave{a} 10$ & 1 à 10 & oui & simple \\
\hline
\end{tabular}

\section{TuÝ̇Re Venturi :}

Une idée souvent reprise consiste à placer l'hélice au col d'une tuyère de Venturi (fis. 14). Cette disposition permet, pour une hélice donnée et un vent donné, d'accroître la vitesse de rotation et la puissance ainsi que le rendement aérodynamique, par suppression des pertes marginales. Mais la tuyère nécessaire est lourde et extrêmement côtteuse; elle offre en outre une prise importante au vent, ce qui alourdit considérablement le pylone. L'accroissement de prix excessif fait disparaittre le faible avantage obtenu du côté de la vitesse et de la puissance.
Notons que la tuyère est beaucoup plus efficace sur les hélices à faible nombre de pales que sur les hélices à grand nombre de pales. On peut arriver à doubler la puissance d'une hélice à deux pales par l'adjonction d'une tuyère. Il n'en reste pas moins que le prix de l'ensemble est tout à fait prohibitif.

Or pour les éoliennes, comme d'ailleurs pour toutes les machines utilisant une énergie naturelle dite «gratuite», ce n'est pas le rendement énergétique qui est déterminant mais le prix de revient de l'énergie produite. 


\section{Prise d'ENERgIE a la JANTE:}

En utilisant une roue avec jante, il est possible de prélever l'énergie de la machine dans la région de plus grande vitesse, soit au moyen d'une courroie soit au moyen d'un galet; cette

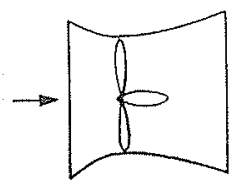

Fig. 14

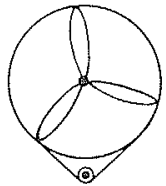

Frg. 15

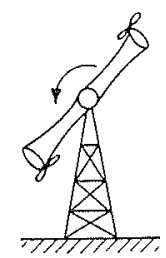

Frg. 16 disposition permet d'éviter l'emploi d'un multiplicateur, mais elle n'est justifiée qu'avec une roue dont la constitution permet la réalisation économique d'une jante (fig. 15).

\section{EOLIENNES EN SÉRIE :}

L'hélice principale comporte à l'extrémité de ses pales des moulinets de petit diamètre entraînant directement des génératrices (fig. 16). Ces moulinets étant attaqués par le vent relatif dont la vitesse est plusieurs fois supérieure à la vitesse du vent, il suffit d'un très petit diamètre pour absorber toute la puissance de l'hélice principale. Ce procédé est ingénieux, mais il nécessite un renforcement considérable des pales de l'hélice principale, dont la forme est d'ailleurs mal adaptée à transmettre l'énergie par son extrémité périphérique. Outre le poids des nacelles d'extrémité, il faut tenir compte d'efforts gyroscopiques importants et d'un accroissement des forces centrifuges. Ces efforts rendent difficile la réalisation de machines à pales orientables. Or, le renforcement de la structure de la pale conduit à l'emploi de profils de grandes dimensions supportant par grand vent une trainée telle que l'effacement des pales s'impose.

\section{Eolienne a dépression :}

L'emploi d'hélice à pales creuses servant de ventilateur aspirant l'air à travers une turbine à air directement reliée à la génératrice constitue également un moyen d'accroître la vitesse d'utilisation sans multiplicateur. Ce dispositif ingénieux a l'inconvénient de présenter un rendement global assez faible. Le rendement global est en effet le produit des rendements de l'hélice, du ventilateur, de la turbine à air et de la génératrice. Pour fixer les idées, le rendement global est environ deux fois plus faible qu'avec une machine à entraînement mécanique. Les pertes les plus grandes se produisent à la sortie des pales creuses à la périphérie, toute l'énergie cinétique de l'air étant perdue. Le faible rendement conduit à augmenter notablement les dimensions de la machine, ce qui fait perdre I'avantage de la simplicité de la transmission. En outre, l'obligation de ménager dans la pale une section suffisante pour le passage de l'air nécessite l'emploi de profils de grandes dimensions et impose par conséquent le montage à pales s'effaçant par grands vents.

\section{Macinine contra-Rotative :}

Cette machine comprend deux hélices tournant en sens contraire et placées l'une devant l'autre. A ces hélices sont fixées des jantes à l'intérieur desquelles se trouvent les éléments de la génératrice électrique. L'une des roues porte l'inducteur et l'autre l'induit. On réalise ainsi une génératrice contra-rotative de grand diametre dont les bras sont les pales de l'éolienne. Cette disposition comporte de grosses difficultés de réalisation; elle est en outre assez peu économique, puisqu'il faut deux hélices pour balayer la mềme aire.

Notons à ce propos que la machine à jante dont il a élé question plus haut peut être prévue de telle facon que la jante constitue le transporteur d'une machine électro-statique, ce qui permet de combiner sans intermédiaire éolienne et génératrice, tout en prélevant l'énergie dans la zone de vitesse maximum.

Il ressort de ce qui précède que le type de machine le plus avantageux pour l'utilisation de l'énergie éolienne el celui que les études de cons. truction feront sans doute ressortir comme le plus économique est le type du genre hélice attaquant directement un axe parallèle à la direction du vent. C'est de ce type uniquement que nous nous occuperons par la suite en laissant de côté toutes les combinaisons et modifications décrites dans les pages précédentes.

Nous aurons à examiner les problèmes suivants :

$1^{\circ}$ réalisation de la roue et des pales;

2 réalisation du pylone;

$3^{\circ}$ orientation dans le vent;

$4^{\circ}$ réglage de la puissance et de la vitesse en fonction du vent;

$5^{\circ}$ transmission de l'énergie de la roue à la génératrice;

$6^{\circ}$ mode d'utilisation.

Nous examinerons ces problèmes uniquement du point de vue de l'exposé et de la classification des différentes solutions qui peuvent leur être apportées, sans nous préoccuper des carac- 
téristiques aérodynamiques ou mécaniques que chaque solution comporte (fig. 17).

Nous sommes maintenant en possession des éléments qui nous permettent de choisir les types et les éléments de machines susceptibles de conduire à des solutions avantageuses. Il est vrai toutefois que la multiplicité des combinaisons est telle qu'on ne peut éviter un certain arbitraire dans le choix des machines. On sera guidé dans ce choix par le souci de satisfaire aux conditions particulières imposées aux éoliennes envisagées.

Dans le cas des éoliennes destinées à débiter sur un réseau, il faut retenir les points suivants :

- puissance relativement importante;

- vitesse constante ou presque constante au récepteur;

- réduction de prix de la génératrice et de la transmission, donc éolienne de type rapide;

- sécurité de marche, même par très grand vent; absence de surveillance;

- simplicité de fabrication; introduire autant que possible des éléments construits en série.

Examinons tout d'abord quelques types de machines dont la simplicité peut séduire.

\section{MOULIN A VENT AMÉLIORÉ :}

Nous entendons par cette désignation une machine proche parente du moulin hollandais à quatre pales dont la rusticité est prouvée, qui fut réalisé à des milliers d'exemplaires avec des dimensions qui, avec un rendement convenable, permettraient facilement d'atteindre une centaine de $\mathrm{kW}$. Le moulin Lykkegaard à persienne constitue une intéressante tentative d'industrialisation du moulin hollandais, mais ses formes, par trop simplistes, limitent considérablement son rendement. Tout en conservant le système de réglage et d'arrêt par persienne, il est possible de donner à la pale un profil convenable, genre aile d'avion, donnant à l'ensemble un rendement moyen très supérieur à celui obtenu avec les ailes non profilées avec un rapport $u / v$ de l'ordre de 3 . Dans ce but, la poutre à section rectangulaire constituant le longeron de la pale est complétée de part et d'autre par un habillage tel que l'on obtienne un profil dont l'épaisseur maximum est égale à l'épaisseur du longeron. On réalise ainsi une aile prismatique à profil et calage constants. L'habillage est constitué par des éléments identiques pouvant, comme les persiennes classiques, pivoter autour d'axes perpendiculaires au longeron (fig. 18). La machine ainsi obtenue est du type à pales fixes. Uń ré- glage grossier peut être effectué en adaptant la position de la persienne à la vitesse du vent. Il semble qu'il soit possible, dans ces conditions, de construire des machines pouvant atteindre $100 \mathrm{~kW}$ et entrainant par multiplicateur des génératrices asynchrones. L'extrapolation à des puissances plus élevées soulèverait toutefois des problèmes de construction de pales et ferait ressortir l'inconvénient de vitesses de rotation malgré tout assez faibles.

\section{Machine a volue :}

L'intérêt de cette machine paraît résider dans une construction très économique de la roue, une certaine facilité de réglage, et un comportement favorable aux grands vents; mais l'examen de différentes dispositions possibles de voiles montre qu'il est très difficile avec ce genre de machine de dépasser la valeur $(u / v)=2$ par suite de la courbure que prend la voile, courbure entraînant un profil défavorable et une mauvaise torsion. Ce type de machine sera donc très lent. Enfin la réalisation d'une roue de grand diamètre conduit à un fractionnement de la voile qui complique la construction. Il paraît difficile de résoudre ces difficultés, surtout pour des machines d'une puissance élevée.

\section{Machines a pales creuses :}

Ce genre de machine est assez séduisant au premier abord par suite des possibilités qu'offre son procédé de transmission entre la roue et la génératrice.

L'avantage de la suppression du multiplicateur, toutefois, se paye chèrement par un accroissement du diamètre de la roue dù au mauvais rendement de l'ensemble. La cause principale du mauvais rendement est la perte de l'énergie cinétique de l'air évacué en bout de pales, énergie qui ne peut être récupérée.

Il semblerait avantageux d'utiliser une telle machine avec une roue à pales fixes tournant au fil du vent en reportant la régulation de vitesse sur la turbine à air de petite dimension. Il faudrait alors prévoir un dispositif évitant l'emballement de la roue et construire cette dernière de telle façon qu'elle puisse résister aux plus grands vents. Pour une machine à pales fixes à très grande vitesse spécifique et pale très étroite, on peut admettre que la roue supporte à l'arrêt les plus grands vents. Pour les roues à pales creuses par contre, il faut prévoir un dispositif d'éclipsage par grand vent, car les pales sont de largeur assez grande à cause du canal qu'elles contiennent à leur intérieur. Ceci milite en faveur de la pale orientable (solution adoptée pour la machine construite en Angleterre). Mais si on accepte la présence du mécanisme d'orien- 
tation des pales, autant monter ce mécanisme sur une éolienne classique à pales orientables dont l'excellent rendement permettra de réduire considérablement les dimensions.

Finalement, en dépit de l'originalité de la solution, on ne voit pas d'avantage décisif à l'emploi d'une machine à pales creuses, pour la production de courant électrique. La technique actuelle des multiplicateurs à engrenages permet d'équiper sans difficulté les éoliennes rapides pour les puissances envisagées. La machine à pale creuse, par contre, peut présenter un réel intérêt si l'énergie désirée doit se présenter sur forme de pression d'air. Accouplée à des installations de climatisation ou de réfrigération, par exemple, elle est tout indiquée.

Passons maintenant à l'examen de solutions mieux adaptées à l'équipement de génératrices électriques de puissance élevée. Pour fixer les idées, cette puissance sera de l'ordre d'une ou plusieurs centaines de $\mathrm{kW}$. Il parait encore prématuré de fixer d'une façon certaine la valeur de la puissance constituant l'optimum économique, cet optimum dépendant des conditions Iocales de vent et du mode de fabrication adopté pour ce type (construction en grande ou petite série, ou à l'unité). Néanmoins dans l'état
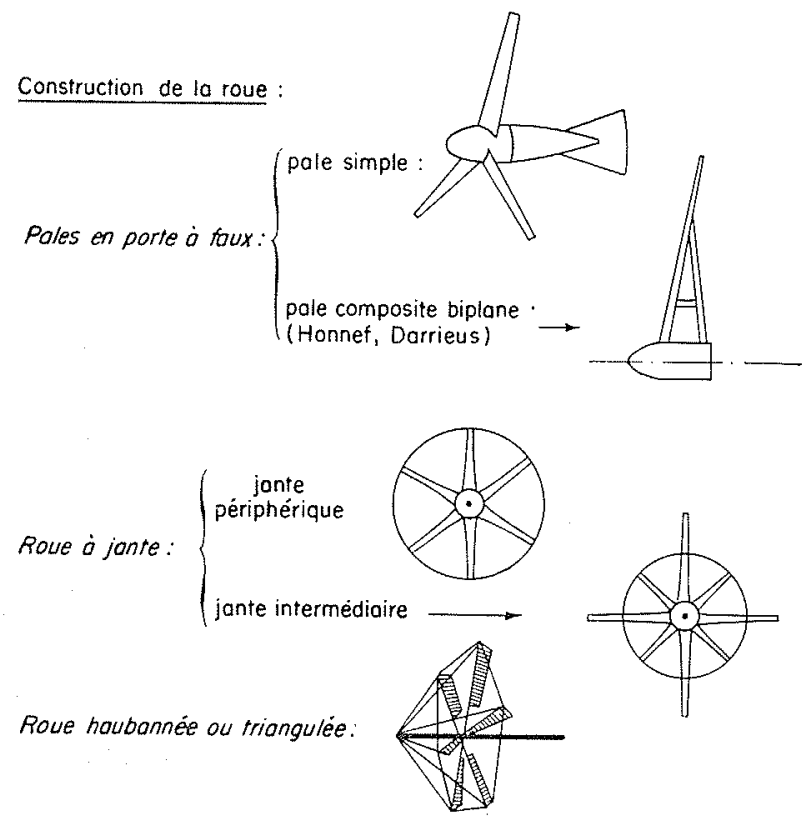

FIG. 171

Construction des poles:

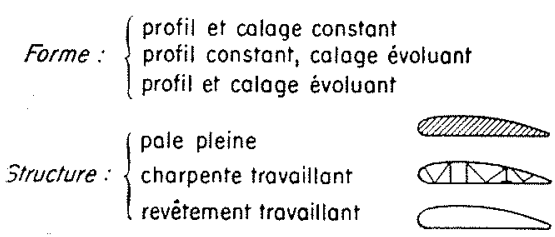

Fig. 172
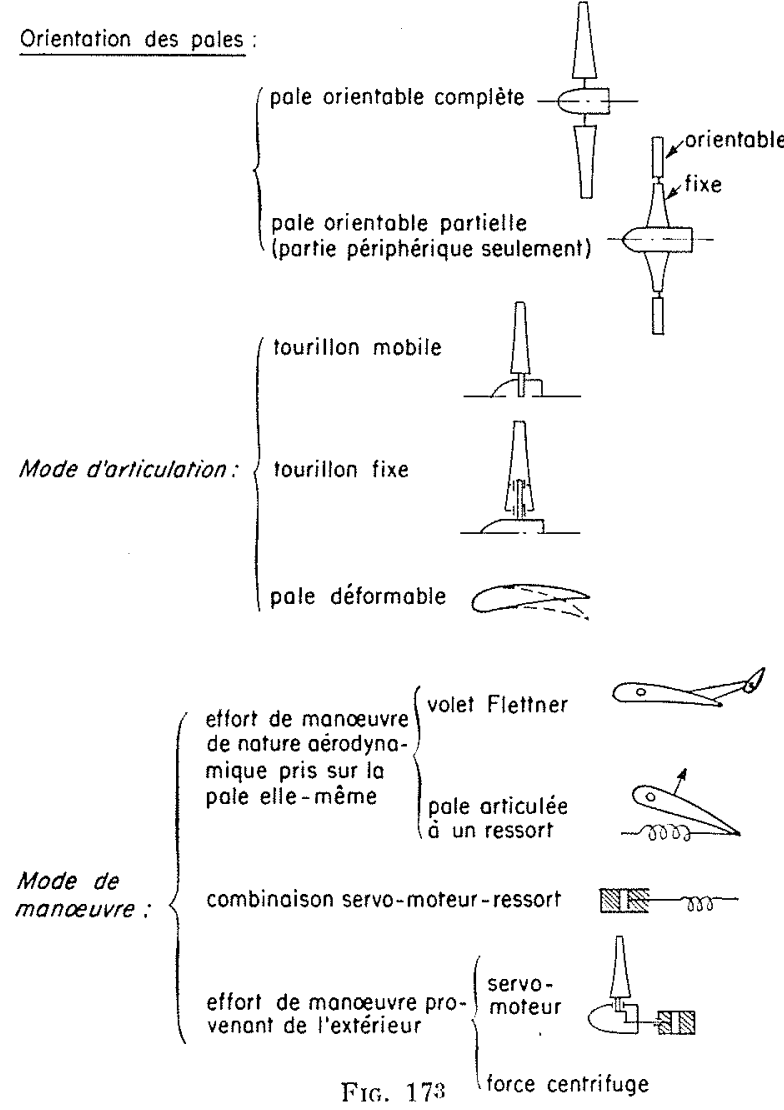

Construction du pylone

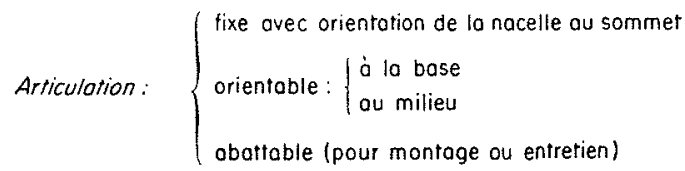

Mode de construction: $\left\{\begin{array}{l}\text { moçonnerie } \\ \text { treillis mérollique } \\ \text { fube }\end{array}\right.$

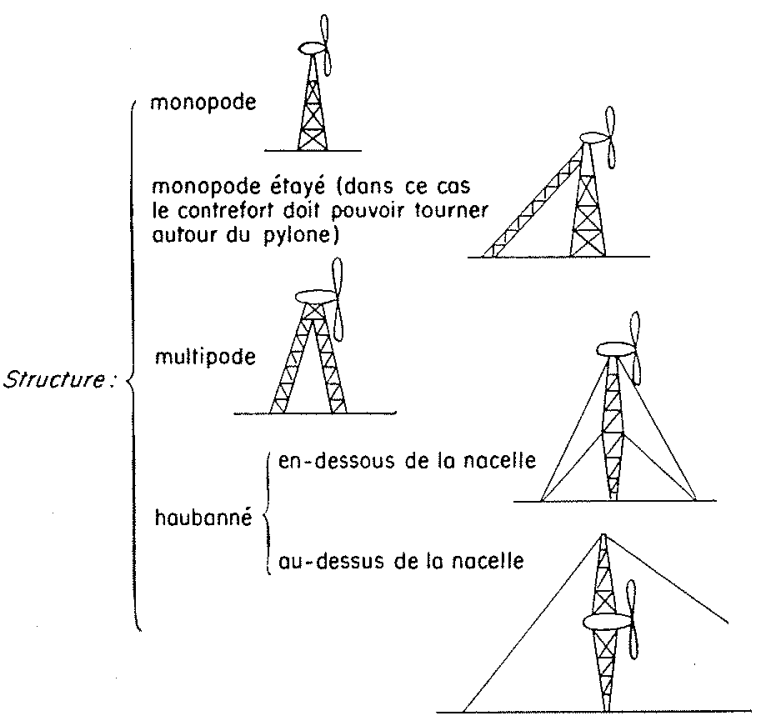

Fra. 174 


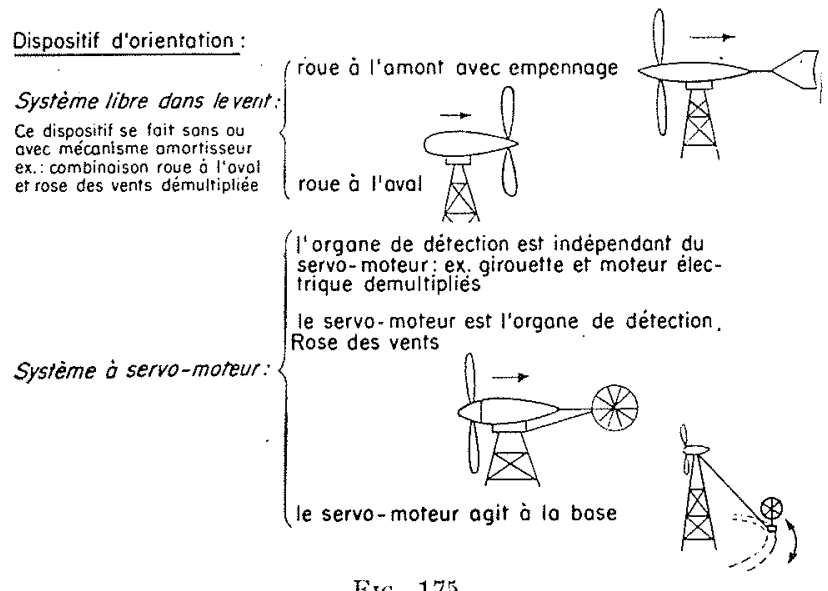

FIG. 175

Régloge:

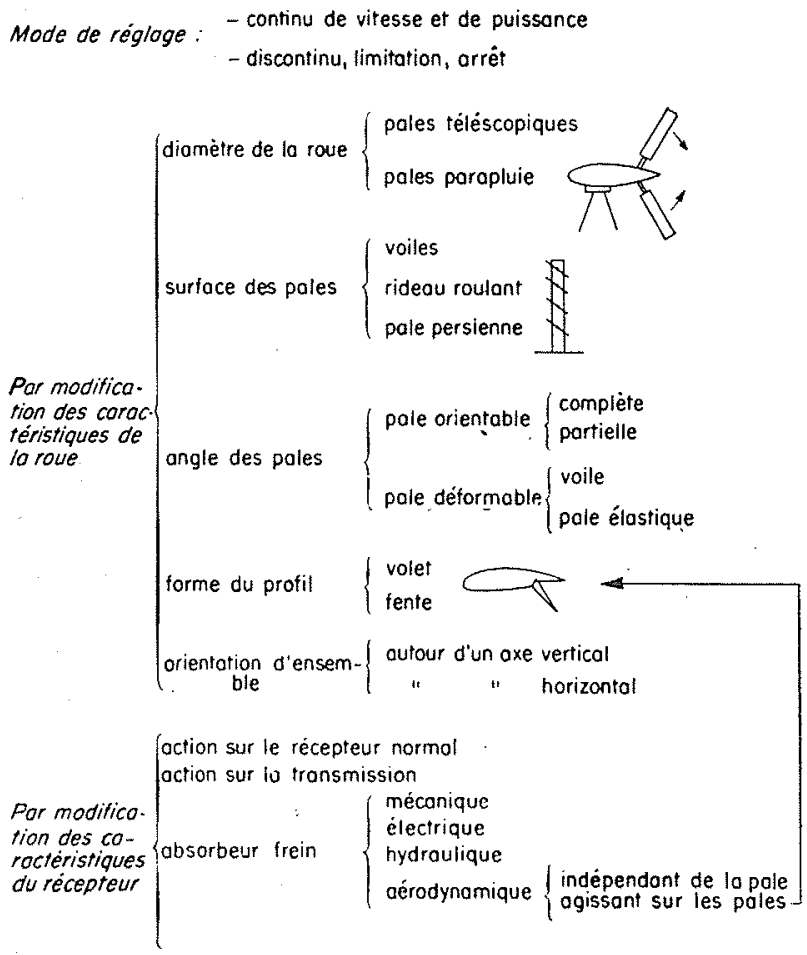

FIG. 17ij

actuel des choses, des machines d'une puissance de l'ordre de $100 \mathrm{~kW}$ doivent apporter un enseignement utile et constituent l'étape nécessaire à franchir avant de se lancer dans la construction de machines plus puissantes, si d'ailleurs il y a un intérêt économique à construire de telles machines.

Deux dispositions de principe s'affrontent: la machine à pales fixes at la machine à pales orientables.
Transmission entre roue et récepteur:
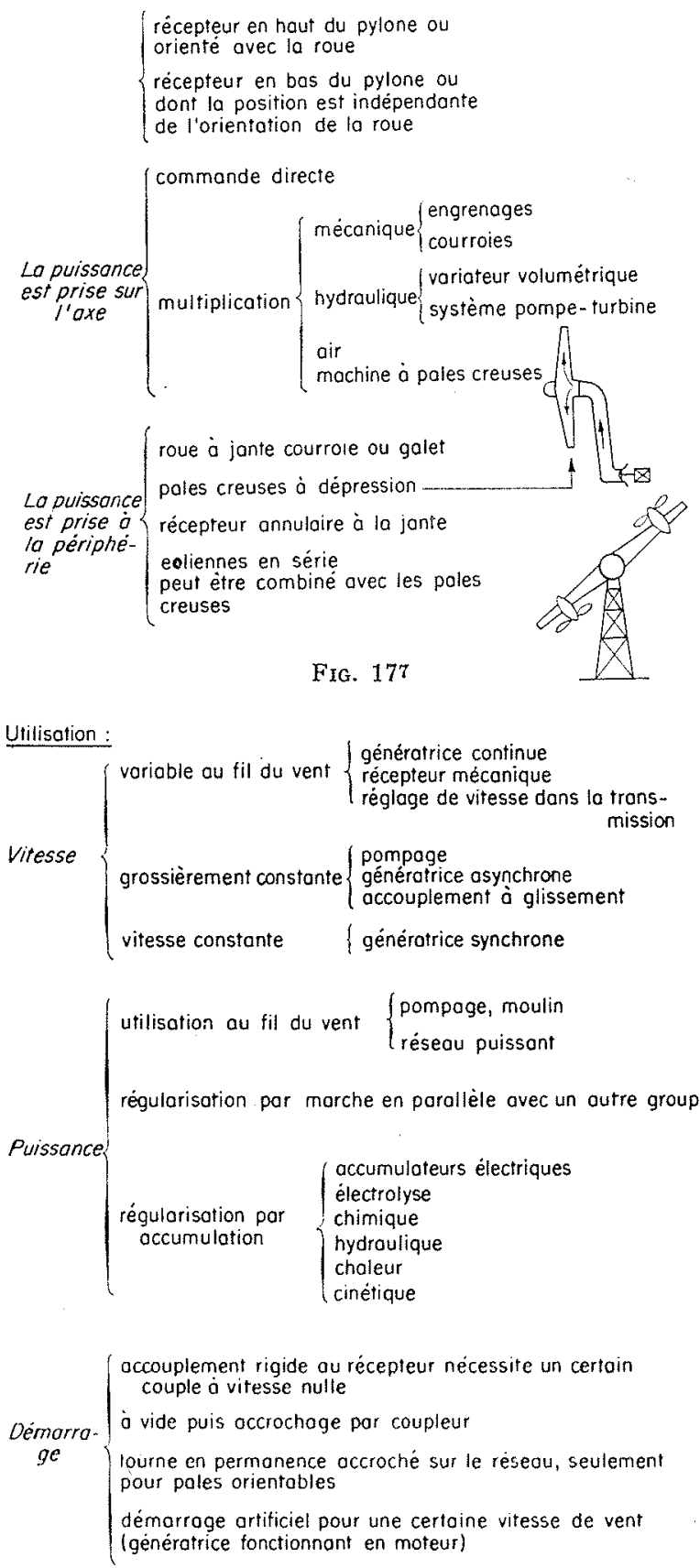

FIi. 178

\section{MACHINE a PALISS FIXTES :}

En l'absence de toute précaution spéciale une telle machine accouplée à une génératrice électrique verrait sa puissance et sa vitesse de rotation crô̂tre continuellement avec la vilesse du venl, ce qui est inadmissible. Pour les machines de petite puissance, on limite vitesse et puissance par des procédés comme l'éclipsage de la roue ou l'utilisation de frein aérodynamique. L'éclip- 


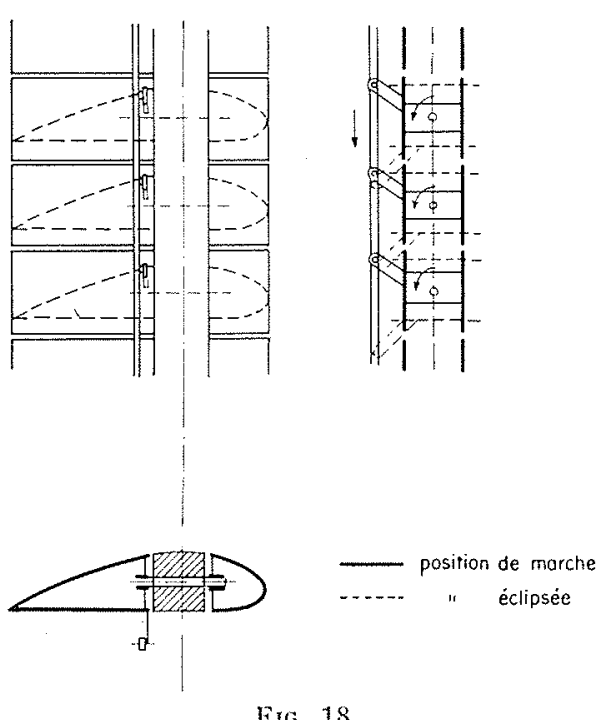

Fig. 18

sage ne saurait être retenu pour des machines de grande puissance, ne serait-ce qu'à cause des importants efforts gyroscopiques qu'il ferait apparaître. Par contre, une intéressante propriété des profils fins à grande vitesse spécifique peut être utilisée. Les polaires de tels profils présentent au voisinage de la portance maximum une brusque baisse de portance due au décollement des filets d'air sur l'extrados. Il en résulte que, si on choisit judicieusement les incidences de fonctionnement, il est possible de réduire considérablement le rendement de la machine à partir d'une vitesse de vent et de limiter la croissance de la puissance lorsque la vitesse du vent augmente. Dès lors, si la machine est accouplée a une génératrice convenable lice au réseau, on peut obtenir une marche à vitesse constante (au glissement près) sans faire apparaître de surpuissance par trop considérable. Un dispositif doit être prévu pour limiter la vitesse d'emballement en cas de disjonction du réseau. Le plus simple est sans doute le frein aérodynamique, sous la dépendance d'un système tachymétrique et qui, placé en bout de pale, permet d'absorber la puissance de l'hélice avec une surface très petite. Le même dispositif peut d'ailleurs être également utilisé pour limiter la puissance en le mettant sous la dépendance d'un système wattmétrique. La machine étant à faible surface de pale et grande vitesse spécifique possède un couple de démarrage si faible qu'il peut être nécessaire de prévoir le lancement, par exemple au moyen de la génératrice utilisée comme moteur. On augmentera grandement la solidité de la rone par l'emploi de pales biplanes ou simplement haubannées, ce qui est possible, puisqu'elles sont fixes; il en résultera une légère baisse de rendement. Il y a intérêt, pour ce type de machine, à placer la roue à l'amont du pylone pour la soustraire à l'influence du sillage de ce dernier; en effet, les pales fixes subissent des fatigues élcrées par suite de la très faible épaisseur relative des profils utilisés. La machine à pales fixes nécessite une génératrice électrique plutôt surdimensionnée. Du point de vue électrique, tout se passe donc comme si on choisissait une puissance nominale correspondant à une vitesse de vent plus élevée que celle qui résulterait de l'utilisation normale de la machine.

\section{Macmine dite a RoUe de BICYCLETTE:}

Isa roue de cette machine comporte une jante périphérique maintenue par des rayons tendus. Chaque rayon constitue une aile dont le profil, de petite dimension, est le même sur toute la longueur du rayon. Ces rayons sont réalisés d'une facon continue par étirage ou par boudinage; ces rayons comportent un corps en matière plastique enrobant une âme constituée par un fil d'acier assurant la résistance à la traction. La jante périphérique est constituée par un assemblage d'éléments standards en tôle roulée. L'ensemble, quoique plus compliqué qu'une hélice classique, doit être d'un prix plus faible par suite de l'emploi exclusif d'éléments de sẻrie.

L'énergie est prise à la périphérie par courroie ou galet de friction; la nacelle contenant la génératrice est placée à la partie inférieure de la roue et s'oriente avec elle (fig. 19). On peut également prévoir une jante conique entraînant par friction un galet conique à axe vertical, monté sur l'axe d'orientation : dans ce cas, la génératrice sera fixe, ce oui simplifie la réalisation de l'ensemble (fig. 20).

Le réglage de vitesse et de puissance pent se faire par éclipsage progressif dans le vent an moyen d'un servo-moteur d'orientation. Suivant une autre disposition simple, le réglage est assuré par torsion des rayons sur eux-mêmes. Dans ce but, les rayons sont fixés rigidement au moyeu, mais reliés à la jante par un dispositif permettant de leur donner une torsion d'un peu plus d'un quart de tour. Ce dispositif comprend, pour chacue rayon, un ressort creux à l'intérieur duquel agit une pression d'huile; ce ressort, rui assure normalement la tension du rayon, pout se déformer sous l'action de la pression d'huile à la manière d'un tube de manomètre métallique. Tous ces ressorts sont reliés à une même canalisation d'huile relice elle-même à l'axe de la roue et alimentée par un petit groupe pompe débitant en circuit fermé sur un orifice calibré el entrainé par une petite éolienne auxiliaire à pales fixes. Lorsque la vitesse du vent augmente, la petite éolienne tourne plus vite, ce qui augmente le débit de la pompe à huile et par suite 


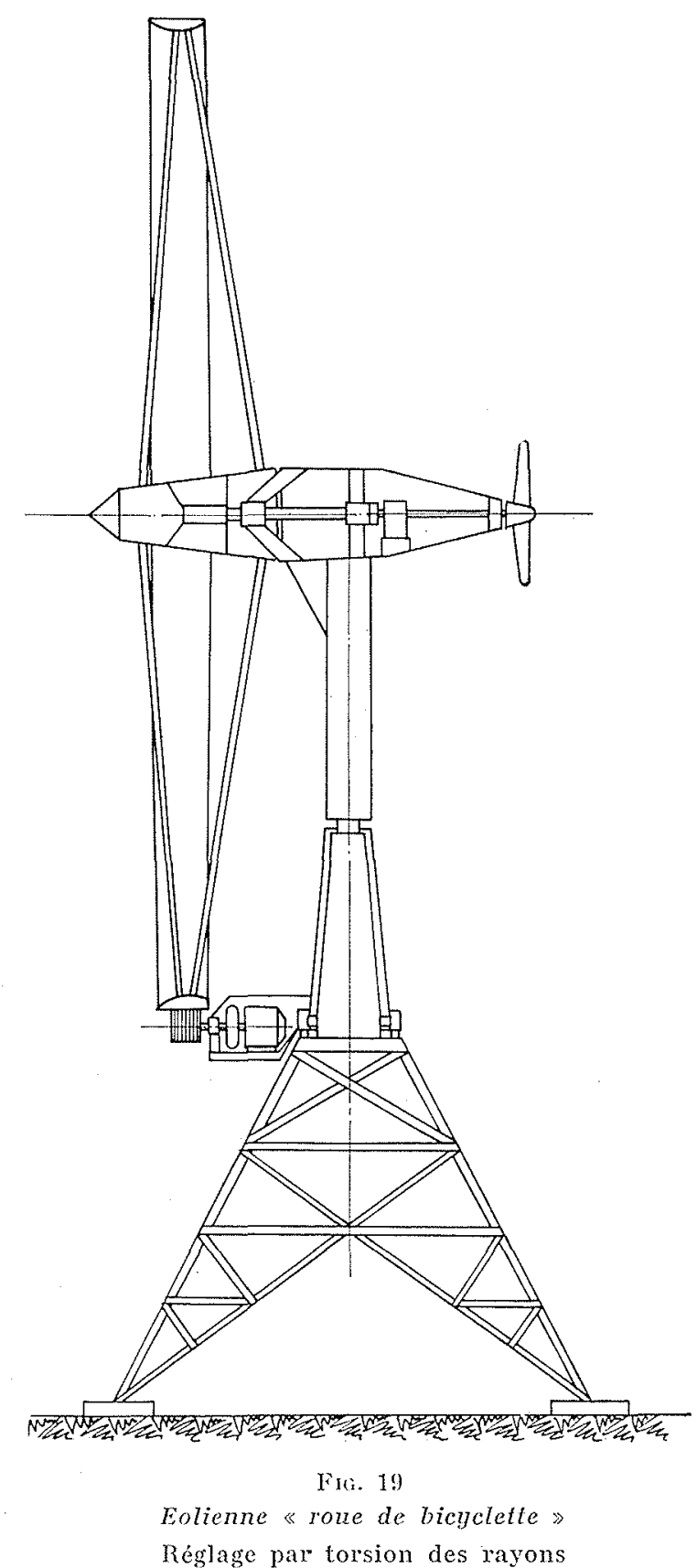

Puissance : $100 \mathrm{~kW}$ (pour un vent de $12 \mathrm{~m} / \mathrm{s}$ sur l'axe, soit $8 \mathrm{~m} / \mathrm{s}$ à $2 \mathrm{~m}$ du sol).

Vilesse: Roue, $45 \mathrm{t} / \mathrm{mn}$; Alternateur, $1.500 \mathrm{t} / \mathrm{mn}$.

$$
u / v=7 \quad \text { nalt }=0,9 \quad \text { Roue }: \mathrm{D}=22 \mathrm{~m}, \mathrm{~h}=20 \mathrm{~m}
$$

augmente la pression. Les ressorts de tension des rayons se dépriment et les rayons sont tordus de telle facon que la vitesse de la roue reste pratiquement constante.

Enfin les profils des rayons peuvent être choisis de telle façon que le réglage et le fonctionnement s'effectuent suivant le principe de la machine à pales fixes, l'originalité résidant alors

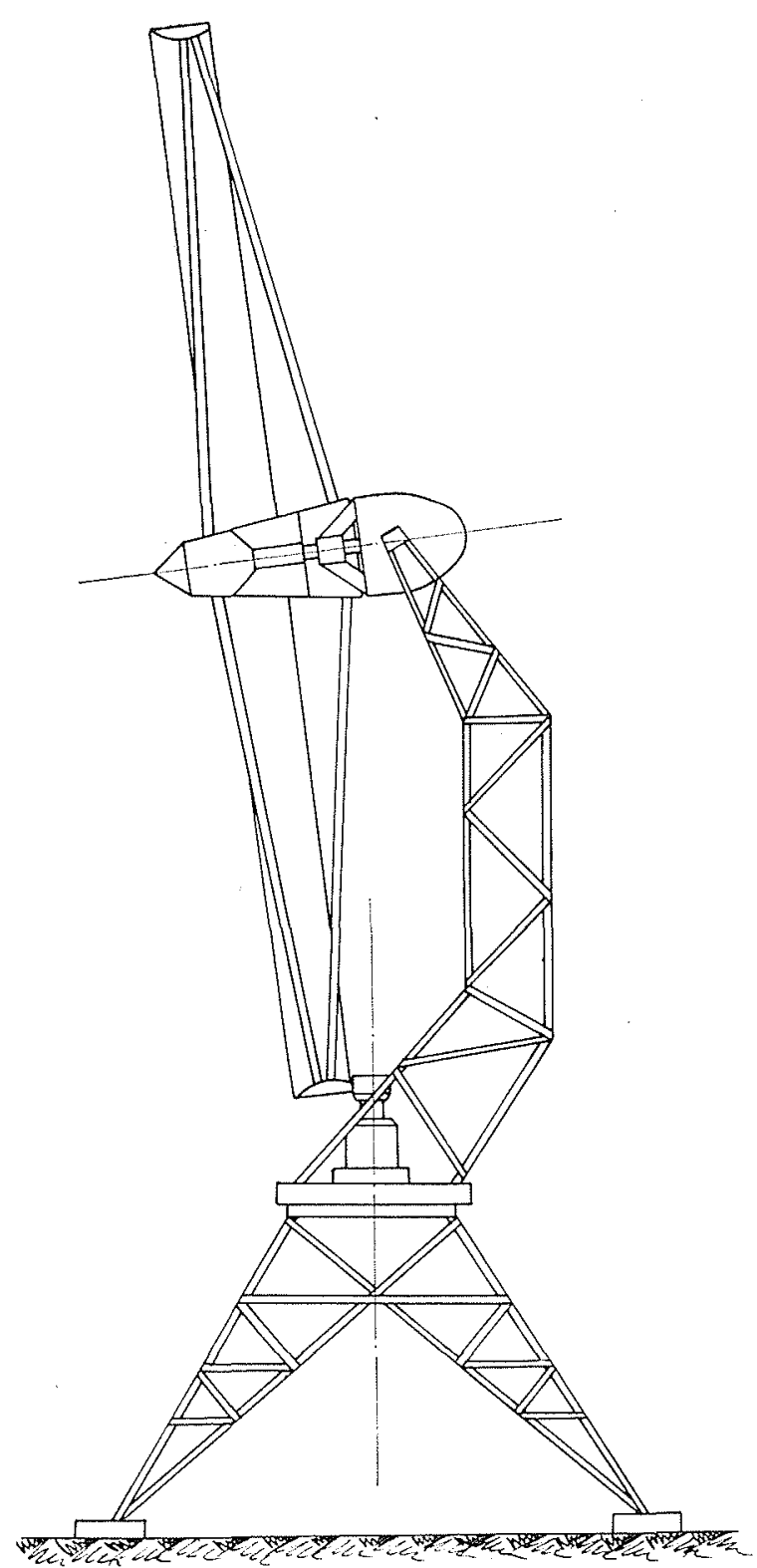

Fig. 20

Eolienne « roue de bicyclette» Alternateur fixe

Puissance: $100 \mathrm{~kW}$ (pour un vent de $12 \mathrm{~m} / \mathrm{s}$ sur l'axe, soit $8 \mathrm{~m} / \mathrm{s}$ à $2 \mathrm{~m}$ du sol).

Vitesse: Roue, $45 \mathrm{t} / \mathrm{mn}$; Alternateur, $1.000 \mathrm{t} / \mathrm{mn}$.

$n / v=7 \quad \eta$ alt $=0,9 \quad$ Roue $: \mathrm{D}=22 \mathrm{~m}, h=20 \mathrm{~m}$

dans le mode de réalisation de la roue et la suppression du multiplicateur à engrenages.

La réalisation de la machine à « roue de bicyclette » pose des problemes nouveaux (transmission par courroie ou par galet, fabrication continue des rayons profilés, etc.); aussi une certaine incertitude subsiste-t-elle sur la possibilité de construction d'une machine de grande puis- 
sance. Il faudra également tenir compte des pertes par frottement de la jante, ce qui entraînera, à puissance égale, une augmentation du diamètre de la rone, de l'ordre de $10 \%$, par rapport aux autres types de machine. Néanmoins l'intérêt de la fabrication en série des éléments constituant la roue, ainsi que la suppression du multiplicateur, suffisent à justifier l'étude approfondie de cet appareil.

Du point de vue du fonctionnement, la machine « à roue de bicyclette » peut être considérée soit comme une machine à pales fixes, soit comme urue machine a pales déformables, donc partiellement adaptée aux variations de vitesse du vent. C'est un type de transition qui nous conduit maintenant aux machines à pales orientables.

\section{Machines a paLes oRIENTABLES.}

Les machines dans lesquelles les pales peuvent pivoter autour de leur axe de tourillonnement, à la manière des hélices d'avion à pas variable, sont très certainement les machines qui permettent le mieux d'adapter la production d'énergie aux variations de la vitesse du vent. Ce sont également celles qui permettent le mieux d'assurer un bon comportement aux tempêtes par mise en drapeau des pales. La difficulté essentielle est de réaliser le tourillon des pales dans une région où s'exerce le moment de flexion le plus important, soit sous les efforts normaux. soit sous les efforts accidentels. C'est par là d'ailleurs que la structure d'une pale d'éolienne peut différer notablement de la structure d'une aile d'avion à laquelle on la compare souvent. Alors que dans l'aile d'avion la section d'encastrement où s'exercent les plus grands moments de flexion est aussi la section la plus grande de l'aile, dans l'éolienne, au contraire, fa section d'encastrement est celle du tomillon, que l'on ne peut accroitre énormément sans augmenter d'autant le prix des paliers et du moyeu. Cette circonstance défavorable est heureusement contre-balancée par le fail que, sur l'éolienne, je poids de l'encastrement peut etre aceru sans inconvénient, ce qui n'est pas le cas de l'aile d'avion. Fn effel, s'il est tris important sur l'éolienne de réduire le moment d'inertie de la roue, par contre, les masses situces au voisinage de l'axe, intervenant peu dans la valeur du moment d'inertie, peuvent être choisies plus grandes, pour assurer la solidité de l'encastrement de la pale. Autre circonstance en faveur de la pale orientable : la possibilité d'ajuster la position de la pale en fonction de la vitesse du vent conduit, à égalité de surface de pale el de vitesse, à des efforts aćrodynamiques plus faibles que ceux obtenus avec la pale fixe et ceci principalement lors des vents de tempête. Dès Jors, la complication mécanique du moyeu et de ses articulations est largement compensée par les avantages d'exploitation. Il ne parait toutefois pas favorable de compliquer outre mesure le mécanisme du moyeu par des dispositifs tels que la mise des pales en parapluie (coning) pour obvier aux rafales de vent. De tels dispositifs, en effet, alourdissent le moyeu, augmentent son prix, peuvent provoquer des vibrations gênantes et sont enfin d'une efficacité problématique.

Etant donné l'intérêt qu'il y a à opérer avec une machine à grande vitesse spécifique, le nombre de pales sera réduit. Pour de très petites machines, on est descendu jusqu'à une seule pale; il semble qu'il s'agisse plus d'une curiosité que d'une solution extrapolable aux grandes puissances. La disposition à deux pales paraît au premier abord plus économique que la disposition à trois pales, mais elle présente le très grave inconvénient d’être génératrice de vibrations lors des mouvements d'orientation, par suite des variations cycliques de l'inertie transversale de la roue (phénomène qui ne se produit plus si le nombre de pales est de 3 ou plus). Pour limiter l'effet de vibration de la machine à 2 pales, on sera conduit à réduire considérablement la vitesse d'orientation de la machine, mais par contre-coup, les efforts transversaux accidentels dus à l'écart entre la direction du vent et l'axe de la roue seront accrus, en sorte que l'économic apparente réalisée par l'emploi de deux pales est absorbée par les renforcements qu'il nécessite. Finalement la disposition à trois pales paraît la plus sûre (fig. 21).

La roue peut être placée en amont ou en aval du pylone. La disposition à l'amont a l'avantage de soustraire les pales à l'effet du sillage du pylone. Notons toutefois qu'indépendamment do leffet du sillage du pylone, chaque pale subit une variation cyclique d'effort par suite de la variation de la vilesse du vent avee la hauteur au-dessus du sol. La disposition à l'amont, dans le cas de pales orientables, augmente le porleà-faux de la roue pour éviter que les pales mises en drapean ne heurtent le pylone. En outre. elle augmente considérablement les efforts nécessaires bour assurer l'orientation de la rone. Aussi sera-t-il plus favorable d'adopler la disposition a l'aval du pylone; on se contentera de réduire le sillage de ce dernier par une construction appropriée.

Pour assurer la commande des pales et leur donner le calage convenable, plusieurs dispositifs peuvent être prévus. Le plus simple comprend un systeme de ressorts tel que, pour chaque valeur de la vitesse du vent, les pales prennent une position d'équilibre assurant le réglage de la puissance et de la vitesse. Ce dispositif seul ne permet pas d'obtenir un réglage 
suffisamment précis pour l'entrainement d'une génératrice synchrone, mais en liaison avec un traînement de génératrice asynchrone ou de génératrice continu. Ce dispositif se comporte particulièrement bien vis-à-vis des rafales. Un autre système consiste à commander les pales par un servo-moteur classique sous la dépendance d'un régleur. Nous allons examiner plus en délail les deux solutions.

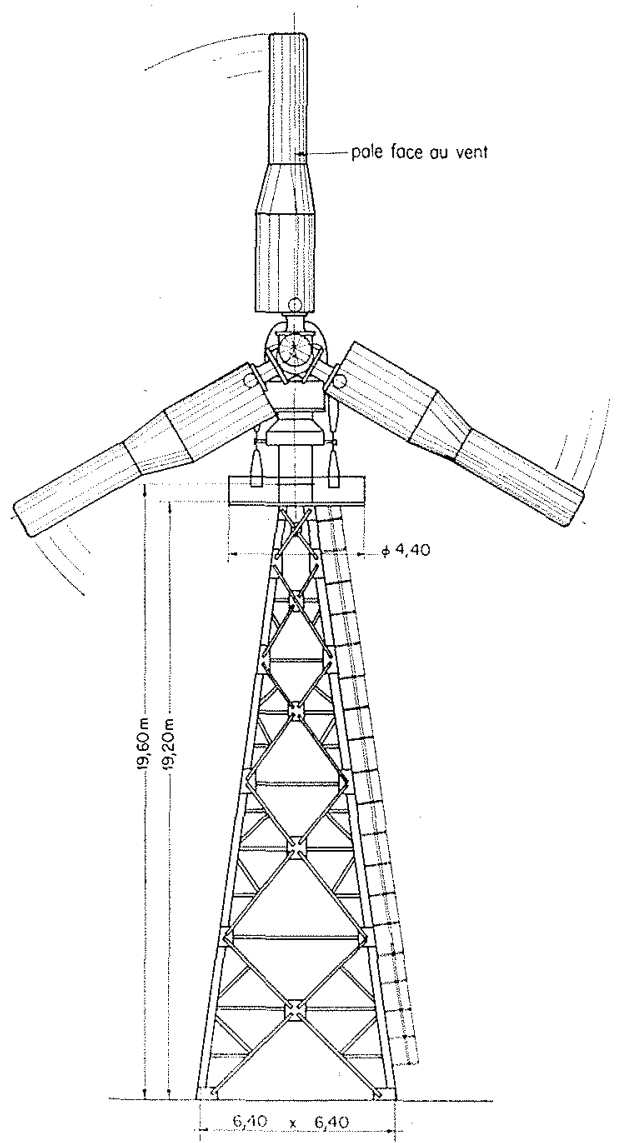

Fia. 21. - Eolienne a pales orientables.

Commande par servo-moteur ou pales a liaison élastique.

Puissance : $100 \mathrm{~kW}$ - - Vilesse : Roue, 60 t/mn. Allernateur, $1.500 \mathrm{t} / \mathrm{mn}$.

Roue : $\mathrm{j})=20 \mathrm{~m} ; \mathrm{h}=24 \mathrm{~m}$. choix concevable des profils, elle permet l'en-

tation soit approximativement constante dans la zone d'utilisation normale. Pour les grandes vitesses de vent, le choix de la caractéristique du système élastique est tel que la vitesse de rotation tend à diminuer el la pale se met en drapeau. Le moment d'incrtie d'une pale autour de son axe de tourillonnement étant relativement faible, le système élastique cède immédiatement sous l'effet d'une rafale et les efforts sur l'ensemble de la construction sont très réduits.

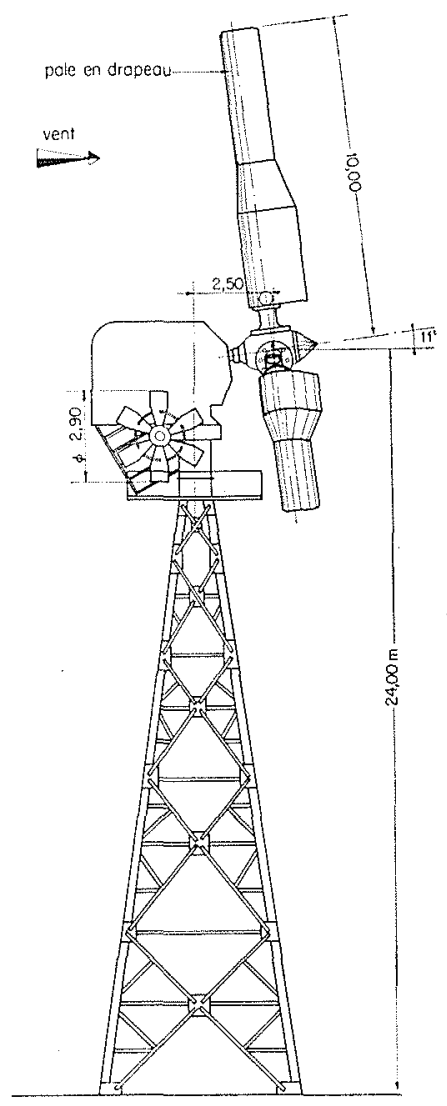

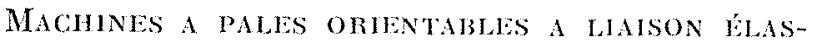
TIQUE.

Dans ce type de machines à pales orientables, l'axe de tourillonnement des pales est choisi de telle manière que la résultante aérodynamique des efrorts sur la pale crée un couple toujours de même signe. Ce couple, croissant avec la vitesse du vent, est équilibré par un système élastique. Dans ces conditions, à chaque valeur de la vitesse du vent correspond une orientation de la pale. On choisit la caractéristique du système élastique de telle manière que la vitesse de ro-
Cette disposition est considéablement plus efficace que celle utilisant le « coning pour assurer l'effacement des pales lors d'une rafale. En effet, dans la machine à pales élastiques, la pale s'efface en pivotant autour de l'axe pour lequel le moment d'inertie est minimum, alors que dans le « coning », cet effacement s'opère autour d'un axe pour lequel le moment d'inertie est maximum. Le mode d'articulation des pales de la machine à pales élastiques contribue à donner à ce genre d'éolienne une grande souplesse qui se traduit finalement par une économie sur le prix de la construction. 
L'intérêt de la disposition à « pales élastiques » est bien mis en évidence par la comparaison des efforts obtenus sur ce genre de construction avec ceux auxquels serait soumise une éolienne à pales fixes.

La comparaison porte sur une machine à 3 pales de $20 \mathrm{~m}$ de diamètre, les pales étant identiques dans tous les cas. On indique dans le tableau suivant, en fonction de la vitesse du vent, les valeurs du moment de flexion $\mathrm{M}_{f}$ de la pale a l'encastrement au moyeu et de la poussée axiale $P_{a r}$ sur l'ensemble de la roue. Ces valeurs sont domnées pour une machine à pales fixes en marche normale, pour cette même ma- jouant le rôle d'anémomètre. Ce mode de réglage est très délicat, car d'une part l'organe détecteur mesure la vitesse du vent en un point et à un instant donnés, d'autre part la machine fournit une énergie qui dépend de la vitesse moyenne du vent traversant la zone balayée par l'hélice. Des écarts considérables peuvent apparaître entre ces deux grandeurs par suite de la turbulence. On peut, dans une certaine mesure, éviter ces difficultés en donnant à l'éoliemne anémomètre une inertic mécanique convenable. Mais, néanmoins, la machine devra être assez largement dimensionnée pour supporter les efforts dus à l'écart de réglage.

\begin{tabular}{|c|c|c|c|c|c|c|c|}
\hline VI'TESSE DU VENT & $7,5 \mathrm{~m} / \mathrm{s}$ & $10 \mathrm{~m} / \mathrm{s}$ & $15 \mathrm{~m} 11 / \mathrm{s}$ & $20 \mathrm{~m} / \mathrm{s}$ & $30 \mathrm{~m} / \mathrm{s}$ & $40 \mathrm{~m} / \mathrm{s}$ & $50 \mathrm{~m} / \mathrm{s}$ \\
\hline Machine a pales fixes, marche normale. & $\begin{array}{l}\mathrm{H}_{\mathrm{i}}=580 \mathrm{~kg} / \mathrm{m} \\
\mathrm{P}_{1, K}=638 \mathrm{~kg}\end{array}$ & $\begin{array}{l}1.025 \\
1.125\end{array}$ & $\begin{array}{l}2.300 \\
2.530\end{array}$ & $\begin{array}{l}4.070 \\
4.170\end{array}$ & & & \\
\hline Machine à pales fixes, roue arrêtée... & $\left\{\begin{array}{l}\mathrm{M}_{t}=108 \mathrm{~kg} / \mathrm{m} \\
\mathrm{P}_{t, x}=131 \mathrm{~kg}\end{array}\right.$ & $\begin{array}{l}193 \\
231\end{array}$ & $\begin{array}{l}430 \\
518\end{array}$ & $\begin{array}{l}765 \\
920\end{array}$ & $\begin{array}{l}1.730 \\
2.070\end{array}$ & $\begin{array}{l}3.030 \\
3.650\end{array}$ & $\begin{array}{l}4.720 \\
5.720\end{array}$ \\
\hline $\begin{array}{l}\text { Machine à pales élastiques, marche nor- } \\
\text { male } \ldots \ldots \ldots \ldots \ldots \ldots \ldots \ldots \ldots \ldots \ldots \ldots\end{array}$ & $\left\{\begin{array}{l}\mathrm{M}_{f}=580 \mathrm{~kg} / \mathrm{m} \\
\mathrm{P}_{u . x}=638 \mathrm{~kg}\end{array}\right.$ & $\begin{array}{l}600 \\
669\end{array}$ & $\begin{array}{l}695 \\
825\end{array}$ & $\begin{array}{r}905 \\
1.105\end{array}$ & $\begin{array}{l}935 \\
987\end{array}$ & $\begin{array}{l}775 \\
850\end{array}$ & $\begin{array}{l}712 \\
733\end{array}$ \\
\hline
\end{tabular}

chine à pales fixes, roue arrêtée face au vent, et enfin pour la machine à pales élastiques en marche normale.

Le réglage de la machine à pales élastiques a été supposé tel que le dispositif ćlastique commence à jouer à partir de la vitesse du vent $v=7,5 \mathrm{~m} / \mathrm{s}$.

Si on admet que la machine doit être calculée pour résister à un vent de $50 \mathrm{~m} / \mathrm{s}$, on constate que, en gros, les efforts normaux sont cing fois plus faibles sur la machine à pales élastiques que sur la machine à pales fixes de même surface, d'où une réduction du prix de la construction.

\section{Machine a pales orientables a servo-moteur :}

Une telle machine se distingue de la précédente uniquement par le mode de commande des pales. Cette commande sera assurée par un servo-moteur généralement à fluide sous pression, lui-même sous la dépendance d'un régleur suivant une disposition classique. Le régleur peut être lui-même sous la dépendance des variations de vitesse du vent, des variations de vitesse de l'éolienne ou des variations de la puissance fournie par la génératrice.

Dans le premier cas, l'organe détecteur serait constitué par une petite éolienne à pales fixes
Le réglage basé sur la mesure de la vitesse de l'éolienne peut être retenu dans le cas d'entraînement d'une génératrice continu ou d'une génératrice asynchrone à gros glissement à la rigueur, mais il est peu indiqué pour une génératrice synchrone qui tourne à vitesse constante. Dans ce cas, en effet, la vitesse n'est pas une grandeur commode pour détecter les varialions de puissance fournie.

Le réglage basé sur la mesure de la puissance fournie par la génératrice paraît être dans tous les cas le meilleur procédé. Il ne faut pas oublier en effet que, dans une éolienne, la puissance et par conséquent les efforts subis par la machine, varient beaucoup plus vite que la vitesse. On améliorera considérablement la sécurité de fonctionnement de la machine en combinant le système de servo-moteur avec une liaison élastique de la commande des pales. II sera ainsi possible, tout en assurant un réglage convenable de la puissance, de supporter les brusques rafales de vent. Il suffit en effet, surtout aux grandes vitesses de vent, d'une faible variation d'orientation des pales pour modifier considérablement les efforts; ces variations accidentelles peuvent être supportées par les liaisons élastiques, cependant que le réglage moyen est assuré par le servo-moteur et le régleur puissance métrique. 


\section{CONCLUSION}

Parmi l'ensemble des éoliennes possibles, nous avons retenu quatre types de machines qui paraîssent pouvoir être réalisées pour de grandes puissances.

La machine à pales fixes est séduisante par sa simplicité, quoique la réalisation des pales, par suite de la nécessité d'employer des profils fins, soit délicate. Certaines réserves doivent également être faites sur les possibilités de fonctionnement aux grandes puissances.

La machine à « roue de bicyclette », qui peut être traitée soit suivant le principe pale fixe, soit suivant le principe pale partiellement orientable, possède une construction basée essentiellement sur l'utilisation d'éléments profilés peu coûteux. Son étude doit montrer à quel point il est possible de réduire le prix de revient du kWh en jouant sur la simplification des procédés de fabrication.

Les deux machines à pales orientables considé. rées ne diffèrent que par leur mode de réglage, mais l'essentiel de leur construction est identique.

La machine à liaisons élastiques se distingue par son comportement aux rafales. La machine à servo-moteur permet de répondre à toutes les exigences de la production d'énergie électrique sur un réseau; en combinaison avec les liaisons ćlastiques, elle permet également d'assurer une bonne tenue aux rafales et aux tempêtes.

Il est bien entendu que pour toutes ces solutions les études n'ont des chances d'aboutir aux résultats désirés, en particulier la rentabilité de l'énergie éolienne, que dans la mesure où on conserve toujours le souci d'une fabrication économique, sans se laisser entraîner à de coûteux perfectionnements mécaniques ou aérodynamiques. Le prix de revient du $\mathrm{kWh}$, enfin, ne sera porté à une valeur acceptable que si un nombre suffisant d'appareils permet de réduire les prix de fabrication en adoptant des procédés de construction en série.

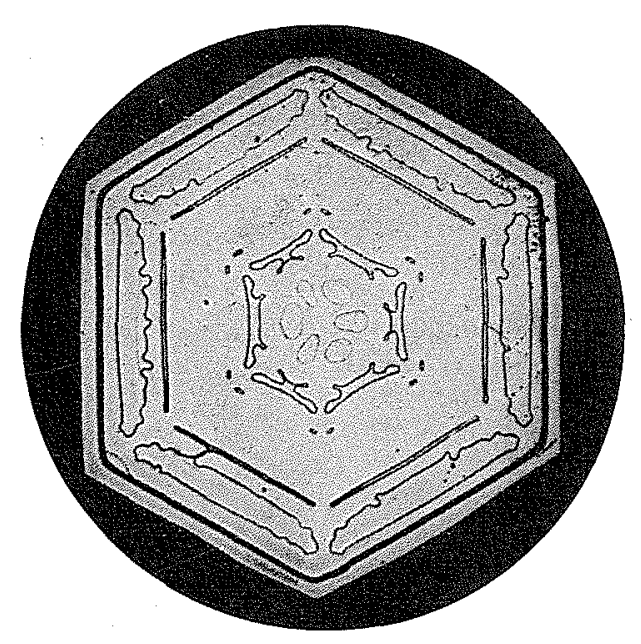




\title{
A Synoptic Study of the Different Types of Windmills
}

\author{
BY I. VADOT \\ INGENIEUR-CONSEI, ATX F́TAILISSTEMENTS NEYRPIC \\ (Translation by E.W. Gommiva)
}

(See French text p. 189 for illustration)

\begin{abstract}
The author begins by a survey of all the possible uays of utilising wind power to drive rotary machinery and then discusses the efficiency and characteristics of each.

The conclusion drawn from this survel is that the only machine of this type that can be applied industrially to a good purpose is the propeller tupe with its axis parallel to the wind direction. The various refinements added to the machine are listed, and suitable designs
\end{abstract}

\begin{abstract}
for their main components-i.e. pylons, wheels, governing, heading control and power transmission sytems-are summarized in a table. Designs likely to be cheap to build and easly to operate, such as improved "Dutch-type" windmills, sail-equipped, hollow-bladed and fixedblade machines, "bicycle wheel" windmills, variable-pitch wheels with elastic linkage or with servo-motors, are discussed in individual paragraphs.
\end{abstract}

At the beginning of the development of a technique when the lack of experience makes selection extremely difficult, a large number of possible types of machines present themselves to the investigator.

Although our experience with small-power machines has already enabled us to propose certain arrangements and to work towards the economic optimum, this is not the case with large-power machines. In this field the number of machines actually constructed is still too small to give sufficiently clear information on the course to pursue.

It is therefore opportune to make a critical study of all possible solutions. We shall then be able to avoid useless retrogression and see in what direction the investigations are most likely to be successful.

A classification of the different methods of converting the energy of the wind into usable energy will show the principal mechanical arrangements whose characteristics and peculiarities will then be examined. As each problem of detail also has several solutions, it will be necessary to review them all critically At the end of this study a certain number of machines will appear, which, for technical and economic reasons, may be considered worthy of interest. It would certainly be premature to indicate at once the most favourable type of machine, even if there were only one, but it will probably be among those which will emerge from this examination.

\section{CLASSIFICATION OF THE DIFFERENT TYPES OF WINDMILLS}

Here we shall consider only machines intended to convert the kinetic energy of the wind into usable mechanical energy on the shaft of the machine driven by it. In all cases, the energy is taken from the wind by means of suitable blades or blade systems moving in a certain direction. Windmills can be placed in two categories according to the direction of displacement of the blade system in relation to the direction of the wind.

(1) Machines in which the rotor blades move in the direction of the wind;

(2) Machines in which the rotor blades move perpendiculary to the direction of the wind. (fig, 1).

\section{Machines of the first type - rotor blades moving in the direction of the wind.}

If $v$ is the wind speed and $u$ the speed of displacement of the propulsive blate system, these machines are characterized by the fact that:

$$
u<v
$$

It can be shown by calculation that their efficien. cy is maximum when $a=v / 3$; these are therefore very slow machines. The axis of the rotor is perpendicular to the direction of the wind; only one part of the blade system is aclive in driving; as the other, on the contrary, is obliged to go against the wind stream, it is neessary to provide a device to reduce the resulting retarding effect. It is this device which characterises the different machines. We can differentiate:

a) Machines with simple drag, in which the blade system going against the wind is withdrawn from its action;

b) Machines with Iras difference, in which the form of the blade is such that its drag is less against the wind than in the direction of the wind. 


\section{a) Machines with simple dRag:}

1. Screen machine: A suitably placed screen eliminates the action of the wind on the blade systems going against the current (fig. 2).

2. Machine of the clapper type: The blades are hinged on axes parallel to the axis of the wheel. A stop placed near each blade holds it back when it sustains the propulsive thrust, but leaves it free to feather in the wind for the return (fig. 3 ).

3. Machine with cyclic variation of blade pitch: By an epicycloidal mechanism the blade turns round its axis at half the speed of the rotor. The effect is analogous to that of the clapper-type machine but without the shocks which occur in the latter (fig. 4).

\section{b) Machines witu drag difterence:}

4. The cup-type windmill is the perfect example of this lind of machine. The shape and the number of cups can obviously vary from one type to another (fig. 5).

5. Transverse-flow machine: These machines constitute a transitional type between Classes I and II. Part of the blade system functions by the difference of dras, but on the other hand a flow of air traverses the machine and works twice in the blade system according to a scheme analogous to that of Banki hydraulic turbines (fig. 6).

6. Savonius rotor: This kind of machine, which derives from the cup type, bears a resemblance to the transverse-flow machine and constitues a clearly transitional type since the peripheral speed may exceed the wind speed in certain cases (fig. 7).

\section{Machines of the second type, rotor blades} moving perpendicularly to the wind.

For this type of machine the speed of displacement $u$ of the rotor may be greater than the wind speed. The thrust on the blade, which in the preceding category was a simple form of drag, is here obtained by deviation of the air stream and is accompanierl by a circulation of the speeds round the profile of the blades. This generating circulation of propulsive thrust is most frequently obtained by the combination of the incidence of the relative current and of the shape of the profile, but it can also be obtained by the use of a device such as the Flettner rotor.

Machines of this class are distinguished by the way in which the rotor is displaced and by the mechanical combinations that this displacement necessitates.

\section{a) "Transverse" MaCHINES:}

7. "Endless belt" machine: In this machine a grid of blades mounted on a system of belts carried by two systems of pulleys moves perpendicularly to the wind. A reversing mechanism of the blades enables a propulsive thrust to be obtained on the two sides. The wind therefore traverses the device twice (fig. 8).

8. Machine with oscillating profile: A wing, the axis of which is perpendicular to the wind, is mounted on a system of rods and cranks out of phase which gives it an oscillating movement with direct thrust first in one direction, and then in the other (fig. 9)

9. Machine with set of rotors: Flettner rotors perpendicular to the wind can move on trucks to which they transmit the motive power. Madaras device) (fig. 10),

\section{b) Machines with cyclic variation of} INCIDENCE:

These machines are also called panemones. They should not be confused with machines of similar appearance operating by drag. From the aerodynamic point of view, they bear a strong resemblance to the endless belt machine. The wind has to traverse the propulsive system twice. This division in two of the propulsive drop leads to a bad utilization of the blades.

10. Panemone with cyclic variation of pitch: In these machines the blade system is propulsive only on the arcs $\mathrm{AB}$ and $\mathrm{CD}$. The wind traverses the blade system twice. The variation of incid. ence on the profiles is here obtained by a suitable rotation of the blades (fig. 11).

11. Fixed-bladed panemone: These machines can be designed only for a speed $u$ which is much greater than the wind speed. The variation of incidence is due to the combination of the speeds; the incidence retains a suitable value only if the speed of the rolor is fairly high, so that it is necessary 10 start the machine in order to initiate its self-operation, or to provide a device giving a sufficient torque for starting. It is possible. with suitable profiles and by blades of conic section, to effect starting by using the drag difference of the profiles (fig. 12).

\section{c) Machines witu axis parathel to thy WIND:}

12. In these machines, the blade system turning round an axis parallel to the wind always has the same orientation in relation to the wind; the operation is therefore perfectly continuous. The shape of the blades may vary considerably; they may consist of Flettner rotors. The speed of displacement of the blades may be much greater than the wind speed (fig. 13).

\section{Value of the different types of windmills}

The essential quality demanded of a windmill is that it should be able to produce the maximum possible energy, in a given wind, and be as economic as possible in design. In order to judge the value of the different machines, we will examine the following points:

1) Speed: The speed will be characterized by the ratio of the tip speed $l$ of the blades to the wind speed $v$. The greater this ratio, the more rapidly the machine will turn, and the lower will be the cost of the parts involved (gearing, generator, etc.).

2) Coefficient of utilization of the blades: This coefficient is the ratio of the effective swept area $S$ to the developed area $S_{a}$ of the blades. Obviously, the greater this ratio, the more economic the machine will be. In the case of machines with their axis parallel to the wind, the area $S$ corresponds to the 
area swept by the blades; on the other hand, in the "drag" machines, this area is distinctly smaller than the swept area since the blade is active in driving only on a fraction of its turn.

3) Mechanical characteristics: Simplicity of construction, necessity of pitch changing in the wind, ctc., are all factors affecting the cost of the machine.

In view of the robust character that a windmill must have to enable it to operate in good conditions without special maintenance, great importance must be attached to the simplicity of the mechanism.

It is also necessary to consider the aerodynamic efficiency of the apparatus. This efficiency, easily evaluated for machines with their axis parallel to the wind, is very difficult to define for machines of the first category. It thus seems more convenient to consider the co-efficient of utilization of the blades, in view of the very general character of the examination envisaged.

The characteristics of the types of machine previously classified are set out inthe following table:

\begin{tabular}{|c|c|c|c|c|}
\hline TYPE OF MACHINE & $\begin{array}{l}\text { TIP-SPEED } \\
\text { RATTO } \\
u / v\end{array}$ & $\begin{array}{c}\text { COEFFT. OF } \\
\text { UTILIZATION } \\
\text { S/S }\end{array}$ & $\begin{array}{l}\text { NEED FOR } \\
\text { PITCH } \\
\text { CHANGING }\end{array}$ & REMARKS \\
\hline 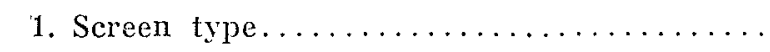 & 0.2 to 0.6 & 0.2 to 0.3 & yes & simple \\
\hline 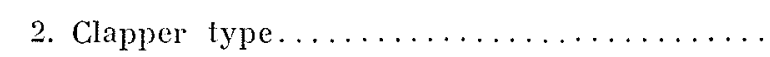 & 0.2 to 0.6 & 0.2 & yes & possible shocks \\
\hline 3. With cyclic variation of blade pitch. & 0.2 to 0.6 & 0.2 & yes & $\begin{array}{l}\text { complicated } \\
\text { mechanism }\end{array}$ \\
\hline 4. Witis drag difference.......... & 0.3 to 0.6 & 0.2 & no & simple \\
\hline 5. Transverse-flow type... & 0.3 to 0.4 & 0.1 & no & simple \\
\hline 6. Savonius .... & 0.5 to 1.5 & 0.5 to 0.8 & no & simple \\
\hline 7. Endless belt. & 2103 & 1 to 2 & yes & $\begin{array}{l}\text { complicated } \\
\text { mechanism }\end{array}$ \\
\hline 8. Oscillating profile............ & 0.5 & 0.5 & yes & $\begin{array}{l}\text { complicated } \\
\text { mechanism }\end{array}$ \\
\hline 9. Set of rotors............ & 2 & 2 to 3 & yes & $\begin{array}{l}\text { necessitates } \\
\text { driving the rotors. } \\
\text { A curiosity. }\end{array}$ \\
\hline 10. Panemone with cyclic variation of pitch. & 2 to 3 & 1 to 2 & yes & $\begin{array}{l}\text { complicated } \\
\text { mechanism }\end{array}$ \\
\hline 11. Fixed-bladed panemone... & 3 to 4 & 2 to 3 & no & $\begin{array}{l}\text { simple, necessitates } \\
\text { starting }\end{array}$ \\
\hline 12. With axis parallel to the wind, propeller type & 1 to 10 & 1 to 10 & yes & simple \\
\hline
\end{tabular}

The above table, based solely on normal operation viously classified are set out in the following table: wind, enables the value of the different types of machines to be estimated.

In the first category (machines in which the rotor blades move parallel to the wind) the machines are

extremely slow and the coefficient of utilization of the blades very low. All these should be rejected. Only the Savonius rotor by its simplicity and the absence of pitch changing mechanism may have some applications for very small power (as a carriage ventilator, for example). 
In the second category the machines are more rapid and have a better coefficient of utilization. The endless-belt type requires a delicate mechanism and an expensive framework difficult to orientate. This machine, like all windmills in which the wind passes twice through the blade system, has a rather low efficiency; the double passage of air prevents its speed from being very high.

The oscillating-profile type is also complicated and difficult to orientate. The machine with a set of rotors is only a curiosity. It will, in fact, be nearly always more advantageous to use wing profiles rather than Flettner rotors.

Panemones with cyclic variation of pitch require a complicated mechanism and also have the faults of the machines in which the wind passes twice through the blade system. Fixed-bladed panemones are an improvement by their simplicity of construction and the absence of pitch changing mechanism, but their speed, coefficient of utilization and efficiency are decidedly lower than for the following type. Moreover, starting is almost always necessary. The simplicity of this apparatus partially disappears if it is necessary to drive a constant speed machine and to consider behaviour in a high wind.

There remains the machine with its axis parallel to the wind (propeller type). The speed attained and the coefficient of utilization of the blades are such that it completely outclasses all the others. It lends itself besides, as will be seen, to numerous variants for the regulation of power and speed. This the other machines do not do, or only badly, and it is an absolutely necessary feature for windmills of a certain size. Finally, it is practically the only one which is able, by means of simple mechanical devices, to stand mp to very high winds.

This rapid examination shows that in the present state of knowledge there are no machines capable of competing effectively with the propeller type.

\section{Modifications made to the propeller type}

Before studying the design of propeller type machines, we will consider some devices for improving them. The principal concern of designers in this field is to increase the speed of rotation of the rotor.

\section{Venturi tuBE:}

An idea often suggested consists in placing the propeller in the throat of a Venturi tube (fig. 14). This arrangement permits, for a given propeller and a given wind, an increase in the speed of rotation and the power, as well as the aerodynamic efficiency, by suppression of the marginal losses. But the necessary tube is heavy and extremely costly and also it presents a large surface to the wind pressure, which results in a considerable increase in the weight of the tower. The excessive increase in cost nullifies the small advantage obtained from the higher speed and power.

Note that the tube is much more effective on propellers with a small number of blades than on those with a large number of blades. The power of a two-bladed propeller can be doubled by the addition of a tube. Nevertheless the cost of the whole equipment is quite prohibitive.
Now for windmills, as of course for all machines which use so-called "free" natural energy, it is not the efficiency which is the decisive factor but the cost of the energy generated.

\section{INTAKE OF ENERGY FROM THE RIM :}

By using a rotor with a rim it is possible to take the energy from the machine in the region or greatest speed, either by means of a belt or of a spur wheel. This arrangement avoids the use of gearing, but it is justified only with a design of rotor which enables the construction of a rim to be economic (fig. 15).

\section{WiNDMILIS IN SERIES:}

The main propeller carries, at the tip of its blades, small windmills driving generators directly (fig. 16). As these small windmills are attacked by the relative wind the speed of which is several times greater than the wind speed, a very small diameter is sufficient to absorb all the power of the main propeller. This method is ingenious but it necessitates considerable reinforcement of the blades of the main propeller the shape of which is also ill-adapted to transmit the energy at its peripheral tip. Besides the weight of the end nacelles, it is necessary to lake into account large syroscopic stresses and an increase of the centrifugal forces. These stresses make the construction of "orientable" or variabli pitch bladed machines difficult. Now the reinforcement of the structure of the blade leads to the use of profiles of large dimensions which: support in a high wind a thrust such that furling of the blades jecomes necessary.

\section{DEPRESSION-TYPE WINDMILI.:}

The use of a hollow-bladed propeller serving as an air pump to draw the air through an air turbine, directly connected to the generator, also provides a means of increasing the speed of utilization without gearing. This ingenious device has the disadvantage of having a rather low overall efficiency. The overall efficiency is, in fact, the product of the efficiencies of the propeller, the air pump, the air turbine and the generator. To give an approximate idea, the overall efficiency is about half that of a machine with mechanical drive. The greatest Iosses occur at the outlet of the hollow blades at the periphery, all the kinetic energy of the air being lost. The low efficiency leads to a considerable increase in the dimensions of the machine, which nullifies the advantage of the simplicity of the transmission. In addition, the necessity of providing in the blade a section sufficient for the passage of the air demands the use of profiles of large dimensions and consequently of blades which will furl in high winds.

\section{COUNTER-ROTATHA MACHINE:}

This machine has two propellers turning in opposite directions and placed one in front of the other. Fixed to them are rims inside which are the parts of the electric generator. One of the wheels carries the rotor and the other the stator. Thus there is a counter-rotating generator of large diameter, the arms of which are the blades of the windmill. This 
arrangement gives rise to considerable difficultics in construction; it is also rather uneconomic since two propellers are necessary to sweep the same area.

Note in this connection that the rim machine mentioned above can be arranged in such a way that the rim constitutes the "transporter" of an electro-static machine, which enables the windmill and generator to be combined without direct coupling while at the same time the energy is extracted in the zone of maximum speed.

It appears from what has been said that the best type of machine for the utilization of wind energy and the one that the design studies will probably show to be the most economic is the propeller type directly driving an axis parallel to the direction of the wind. It is with this type that we shall now be exclusively concerned, neglecting all the combinations and modificalions described in the preceding pages.

We shall have to examine the following problems:

(1) Construction of the rotor and the blades;

(2) Construction of the tower;

(3) Yawing into the wind;

(4) Regulation of the power and speed as a function of the wind;

(5) Transmission of the energy of the rotor to the generator;

(6) Method of use.

We shall examine these problems solely from the point of view of the description and classification of the different solutions which can be applied to them, without considering the aerodynamic or mechanical characteristics involved in each solution (fig. 17).

We have now reached the point where we can choose the types and parts of machines which are likely to be satisfactory. Nevertheless, the multiplicity of the combinations is such that the choice will inevitably be rather arbitrary. We shall be guided by the need to satisfy the particular conditions imposed on the windmills envisaged.

For machines intended to feed into a network, the following points should be borne in mind:relatively large power;

constant or nearly constant speed at the driven machine;

reduction in the cost of the generator and power transmission, therefore a rapid type of windmill; reliability of operation even in a very high wind, absence of supervision;

simplicity of construction, including mass-produced parts as much as possible.

Let us examine first what types of machines may be attractive by their simplicity.

\section{IMPROVED WINDMILL:}

By this is meant a windinill similar to the four.. bladed Dutch windmill whose robustness has been proved. Thousands of them were constructed with dimensions which, with a suitable efficiency, enabled them easily to reach about $100 \mathrm{~kW}$. The slattedtype Lykkegaard windmill represents an interesting attempt to industrialize the Dutch type of windmill, but its over-simplified forms considerably limit its efficiency. While retaining the system of regulation and stopping by slats, it is possible to give the blade a suitable profile, like an aeroplane wing, giving the whole equipment an average efficiency much higher than that obtained with non-profiled wings with a ration $u / v$ of the order of 3 . For this purpose, the beam of rectangular section constituting the spar of the blade is completed on both sides by a fitting such that a profile is obtained the maximum thickness of which is equal to the thickness of the spar. In this way is constructed a prismatic wing of constant profile and stalling. The fitting consists of identical parts which are able, like the orthodox slats, to pivot round axes perpendicular to the spar (fig. 18). The machine thus oblained is of the fixedbladed type. Regulation, though not very fine, can be effected by adapting the position of the slat to the wind speed. It seems possible in these conditions to build machines which can reach $100 \mathrm{~kW}$ and drive asynchronous generators through gearing. Extrapolation at higher powers would, however, raise problems in the construction of the blades and would emphasize the disadvantage of speeds of rotation which are inevitably rather low.

\section{SAIL MACHINE:}

The advantage of this machine seems to be in a very economical construction of the rotor, a certain facility of regulation and favourable behaviour in high winds, but an examination of different possible arrangements of sails shows that it is very difficult to exceed the value $u / v=2$ as a result of the curvature taken by the sail-curvature producing an unfavourable profile and a bad torque. This iype of machine would, therefore, be very slow. Finally, the construction of a rotor of large diameter is complicated because it necessitates the splittings up of the sail. It appears difficult to solve these problems, especially for high-power machines.

\section{Hollow-Bladed Machine:}

This kind of machine is rather attractive at first sight on account of the possibilities offered by its method of power transmission between the rotor and the generator.

The advantage of eliminating the gearing is , however, more than offset by an increase in the diameter of the rotor due to the low efficiency of the whole equipment. The principal cause of the low efficier$\mathrm{cy}$ is the loss of the kinetic energy of the air evacuated at the end of the blades-energy which cannot be recovered.

It would seem advantageous to use such a machine with a fixed-bladed rotor turning with the wind stream and to transfer the speed regulation on to the small air turbine. It would, therefore, be necessary to provide a device to prevent the rotor running light and to construct the latter in such a way that it could resist the strongest winds. Fo? a fixed-bladed machine at very high specific speed and very narrow blades, it may be said that the rotor withstands, at slandstill, very high winds. For rotors with hollow blades, on the other hand, it is necessary to provide a spilling device in a high wind because the blades are rather wide. This makes the variable-pitch blade more favourable (the solution adopted for the machine built in England). But if there is no objection to the use of pitch-changing mechanism, it can be mounted 
on a classic windmill with variable pitch blades and the high efficiency of this windmill will enable the dimensions to be considerably reduced.

Finally, in spite of the originality of the idea, ihere seems to be no decided advantage in the use of a hollow-bladed machine for the generation of electricity. Modern gearing enables high-speed windmills to be equipped without difficully for the powers envisaged. Nevertheless, the hollow-bladed machine may have a real advantage if the power is required in the form of air pressure. Connected to air-conditioning or refrigerating installations, for example, it is highly satisfactory.

We come now to the examination of projects better adapted to the equipment of high-power electric generators. To enable one to form an idea, this power may be of the order of one or several hundred kilowatts. It still seems premature to fix definitely the value of the power which would constitute the economic optimum because this optimum depends on local wind conditions and on the methor of manufacture adopted-production on a large or small scale, or singly. Nevertheless, in our present state of linowledge, machines of a power of the order of $100 \mathrm{~kW}$ will give us useful information and are a necessary stage to pass through before we begin the construction of more powerful machinesif, in any case, there is any economic advantage in constructing such machines at all.

Two main types confront each other--the machine with fixed blades and the machine with variable. pitch blades.

\section{MACHNE WITH FIXED BLADES:}

In the absence of any special precaution, such a machine connected to an electric generator would have its power and its speed of rotation continuously increased with the speed of the wind. This is inadmissible. For small power machines speed and power are limited by methods like turning the rotor out of wind or the use of an air brake. Turning out of wind is impracticable for large-power machines, if only because it gives rise to large gyroscopic stresses. On the other hand, an interesting property of fine profiles at high specific speed can be used. The polars of such profiles present, in the neighbourhood of maximum lift, a sharp reduction of lift due to the release of air streams on the extrados. As a result, if the pitch of operation is chosen carefully, it is possible to reduce considerably the efficiency of the machine at a certain wind speed and to limit the increase of the power when the wind speed increases. Consequently, if the machine is coupled to a suitable generator connected to the network, operation at constant speed can be obtained (slip excepted) without too much overpower occurring. A device must be provided to limit the speed when running light if the machine becomes disconnected from the network. The simplest is undoubtedly the air brake controlled by a tachometric system. This, placed at the tip of the blade, enables the power of the propeller to be absorbed with a very small surface. The same device can also be used to limit the power by controlling it with a wattmetric system. As the machine has a small blade area and high specific speed, the starting torque is so low that starting may have to be effected by means of the generato: used as a motor. The solidity of the rotor is greatly increased by the use of biplane blades or blades simply guyed. This is possible since the blades are fixed, but will result in a slight reduction of efficiency. For this type of machine, there is an advantage in placing the rotor up-wind of the tower in order to remove it from the influence of the wake of the tower; in fact, fixed blades are subjected to high fatigues as a result of the very small relative thickness of the profiles used. The fixed-bladed machine necessitates an electric generator rather over-dimensioned. From the electrical point of view, it is as if one had chosen a rated power corresponding to a higher wind speed than that which would result from the normal utilization of the machine.

\section{"Bicycle WheEl" Maciane:}

The wheel of this machine consists of a peripherat rim maintained by taut spokes. Each spoke constitutes a wing of which the profile, of small dimension, is the same on the whole length of the spoke. These spokes are made continuously by wire drawing or twisting; they consist of a plastic exterior covering a core of steel wire ensuring resistance to traction. The peripheral rim is constructed by jointing standard parts of rolled sheet iron. The whole equipment, although it is more complicated than a traditional propeller, must be cheaper as a resull of the exclusive use of mass-produced parts.

The energy is taken at the periphery by belt or friction roller; the nacelle containing the generator is placed at the lower part of the wheel and turns with it (fig. 19). It is also possible to provide a conical rim driving by friction a vertical axis conical roller mounted on the pintle shaft; in this case the generator will be fixed, which simplifies the construction of the whole apparatus (fig, 20).

The speed and power may be regulated by progressive spilling in the wind by means of a yawing servo-motor. According to another simple arrangement, regulation is assured by twisting the spokes on themselves. For this purpose, they are fixed rigidly to the hub but connected to the rim by a device enabling them to be given a twist of a little more than a quarter of a revolution. This device consists, for each spoke, of a hollow spring inside which an oil pressure operates; the spring, which normally maintains the tension of the spoke, can be lwisted under the action of the oil pressure in the manner of a metal manometer tube. All the springs are connected to the same oil pipe, which is itself connected to the main shaft and supplied by a small pumping set delivering in a closer circuit to a calibrated opening and driven by a small fixed-bladed auxiliary windmill. When the wind speed increases, the small windmill turns more quickly, which increases the delivery of the oil pump and consequently increases the pressure. The tension springs of the spokes become lower and the spokes are twisted in such a way that the speed of the wheel. remains practically constant.

Finally the profiles of the spokes may be chosen in such a way that control and operation are carried out according to the principle of the fixed-bladed machine, the originality then residing in the manner 
of constructing the wheel and the elimination of gearing.

The construction of the "bicycle wheel" machine raises some new problems (power transmission by belt or roller, continuous manufacture of sectional spokes, etc.); also there is some uncertainty aboul the possibility of constructing a machine of large power. It would also be necessary to take inti account the frictional losses of the rim, which would involve, with equal power, increasing the diameter by about 10 per cent in comparison with other types of machine. Nevertheless, the adrantage of mass production of the parts constituting the wheel as well as the elimination of gearing are sufficient to justify a thorough study of this apparatus.

From the operating point of view, the "bicycle wheel" machine can be considered either as a machine with fixed blades, or as a machine with "deformable" blades and therefore partially adapted to variations of wind speed. It is a transitional type which brings us now to machines with variable pitch blades.

\section{MACHines With VARIABLe pITCH bLADES:}

Machines in which the blades can revolve round their axis of pitch, in the manner of aeroplane propellers of variable pitch, are most certainly those which best enable the production of energy to be adapted to variations of wind speed. They also give the best protection against storms by the feathering of the blades. The essential difficulty is to place the blade shank in a region where the greatest bending moment is exerted, either under normal stresses or under accidental stresses. It is there that the structure of a windmill blade may differ appreciably from the structure of an aeroplane wing, to which it is often compared. While, in the aeroplane wing, the root section where the greatest bending moments occur is also the largest section of the wing, in the windmill, on the contrary, the root section is that of the shank, which cannot be greatly increased without at the same time increasing the cost of the foot-step bearings and the hub. This unfavourable circumstance is fortunately counter-balanced by the fact that, on the windmill, the weight of the root can be increased without any disadvantage, which is not the case with the aeroplane wing. In fact, although it is very important, on the windmill, to reduce the moment of inertia of the rotor, on the other hand, the weight situated in the neighbourhood of the axis, which has little effect on the value of the moment of inertia, can be increased to strengthen the root of the blade. Another circumstance in favour of the variable pitch blade is that the possibility of adjusting the position of the blade according to the wind speed leads, with equal blade surface and speed, to lower aerodynamic stresses than those which occur with the fixed blade, particularly at times of very high winds. Consequently, the mechanical complication of the hub and of its articulations is largely counterbalanced by the advantages in operation. There does not, however, appear to be any advantage in unduly complicating the mechanism of the hub by devices such as coning to obviate the effect of gusts. Such devices, in fact, increase the weight of the hub and its cost, may cause troublesome vibrations and are of disputable efficacy.
In view of the advantage of operating with a machine of high specific speed, the number of blades would be small. For very small machines, it might be as low as one blade. The design seems to be an interesting curiosity rather than one from which data can be extrapolated for high powers. The design with two blades seems at first sight more economic than that with three blades, but it has the very serious disadvantage of causing vibrations with the rawing movements as a result of the cyclic variations of the transver'se inertia of the rotor (a phenomenon which does not occur if the number of blades is three or more). To limit the effect of vibration with the two-bladed machine, the speed of yawing of the machine would have to be considerably reduced, but, as a result, the accidental transverse stresses due to the divergence between the direction of the wind and the axis of the rotor would be increased, so that the apparent economy obtained by the use of two blades is nullified by the reinforcements that it necessitates. The design with three blades therefore seems to be the best (fig. 21):

The rotor may be placed up-wind or down-wind of the tower. The arrangement up-wind has the advantage of removing the blades from the effect of the wake of the tower. It must be remembered, however, that independently of the effect of the wake of the tower, each blade is subjected to a evelic variation of stress as a result of the variation of the wind speed with the height above ground. With variable pitch blades, the arrangement up-wind increases the overhang of the rotor to prevent the blades when feathered from knocking against the tower. In addition, it increases considerably the stresses necessary to ensure yawing. Consequently, it would be better to adopt the arrangement downwind; a suitable construction would be sufficient to reduce the wake of tower.

To ensure the control of the blades and to give them the appropriate stalling, several devices can be provided. The simplest consists of a system of springs such that for each value of the wind speed the blades take a position of equilibrium which ensures the regulation of the power and of the speed. This device alone does not give sufficiently precise control for driving a synchronous generator, but, combined with a suitable choice of profiles, it enables an asynchronous or direct current generator to be driven. This device behaves particularly well in gusts. Another system consists in controlling the blades by an orthodox servo-motor depending on a governor. We shall examine the two possibilities in more detail.

\section{MACHINE WITH VARIABLE PITCH BLADES WITH FLEXIBL MOUNTING:}

In this type of machine with variable pitch blades the axis of pitch of the blades is chosen in such a way that the aerodynamic resultant of the stresses on the blade creates a torque which is always of the same sign. This torque, increasing with the wind speed, is balanced by a flexible system. In these conditions, to each value of the wind speed there is a corresponding pitch of the blade. The characteristic of the flexible system is chosen in such a way that the speed of rotation is approximately constant in the normal zone of utilization. For 
high wind speets, the choice of the characteristic of the flexible system is such that the speed of rotation tends to diminish and the blade feathers. As the moment of inertia of one blade round its axis of orientation is relatively small, the flexible system yields immediately under the effect of a gust and the stresses on the whole construction are greatly reduced. This arrangement is considerably more effective than the coning of the blades in gust. In fact, in the flexible-bladed machine the blade is furled by revolving round the axis for which the moment of inertia is minimum, while in coning the furling is effected round an axis for which the moment of inertia is maximum. The method of pitch changing of the blades of the flexible-bladed machine helps to make this kind of windmill very adaptable, which results finally in economy on the cost of construction.

The advantage of the "flexible-bladed" arrangement is clearly shown by comparing the stresses obtained on this lind of construction with those to which a fixed-bladed windmill would be subjected.

The comparison relates to a 3 -bladed machine of $20 \mathrm{~m}$. diameter, the blades being identical in each case. The following table shows the values, as a function of the wind speed, of the bending moment $M_{f}$ of the blade where it is embedded at the hub and of the axial thrust $\mathrm{P}_{a: n}$ on the whole of the rotor. These values are given for a fixed-bladed machine in normal operation, for the same fixedbladed machine with the rotor shut down facing into wind, and finally for the flexible-bladed machine in normal operation. solely in the method of controlling the blades. The control will be effected by a servo-motor, generally hydraulic, which is dependent on a governor of a classic type. The governor itself may be dependent on variations of the wind speed, variations of the speed of the windmill or variations of the power supplied by the generator.

In the first case, the sensing mechanism would consist of a small fixed-bladed windmill acting as an anemometer. This method of control is very delicate, for, on the one hand, the sensing mechanism measures the wind speed at a given point and instant, on the other hand, the machine supplies a quantity of energy which depends on the mean speed of the wind traversing the area swept by the propeller. There may be considerable differences between the two values as a result of turbulence. To some extent, these difficulties may be avoided by giving the windmill anemometer is suitable mechanical inertia. Nevertheless, the machine must be of large enough dimensions to support the stresses due to the divergence of control.

Control based on the measurement of the speed of the windmill may be used for driving a direct current generator or an asynchronous generator of large slip, if necessary, but it is not very suitable for a synchronous generator turning at constant speed. In this case, in fact, the speed is not a convenient value to take for detecting the variations of power supplied.

Control based on the measurement of the power supplied by the generator appears to be in all cases the best method. It must not be forgotten, in fact,

\begin{tabular}{|c|c|c|c|c|c|c|c|}
\hline WIND SPEED & $7,5 \mathrm{~m} / \mathrm{s}$ & $10 \mathrm{~m} / \mathrm{s}$ & $15 \mathrm{~m} / \mathrm{s}$ & $20 \mathrm{~m} / \mathrm{s}$ & $30 \mathrm{~m} / \mathrm{s}$ & $40 \mathrm{~m} / \mathrm{s}$ & $50 \mathrm{~m} / \mathrm{s}$ \\
\hline Fixed-bladed machine, normal operation. & $\left\{\begin{array}{l}M_{y}=580 \mathrm{~kg} \cdot \mathrm{m} \\
\mathrm{P}_{a: 2}=638 \mathrm{~kg}\end{array}\right.$ & $\begin{array}{l}1,025 \\
1,125\end{array}$ & $\begin{array}{l}2,300 \\
2,530\end{array}$ & $\begin{array}{l}4,470 \\
4,070\end{array}$ & & & \\
\hline Fixed-bladed machine, rotor shut down.. & $\left\{\begin{array}{l}M_{f}=108 \mathrm{~kg} \cdot \mathrm{m} \\
\mathrm{P}_{\mathrm{t} x: x}=131 \mathrm{~kg}\end{array}\right.$ & $\begin{array}{l}193 \\
231\end{array}$ & $\begin{array}{l}430 \\
518\end{array}$ & $\begin{array}{l}765 \\
920\end{array}$ & $\begin{array}{l}1,730 \\
2,070\end{array}$ & $\begin{array}{l}3,030 \\
3,650\end{array}$ & $\begin{array}{l}4,720 \\
5,720\end{array}$ \\
\hline Flexible bladed machine, normal operation & $\left\{\begin{array}{l}\mathrm{M}_{f}=580 \mathrm{~kg} \cdot \mathrm{m} \\
\mathrm{P}_{u, 3:}=638 \mathrm{~kg}\end{array}\right.$ & $\begin{array}{l}600 \\
669\end{array}$ & $\begin{array}{l}695 \\
825\end{array}$ & $\begin{array}{r}905 \\
1,105\end{array}$ & $\begin{array}{l}935 \\
987\end{array}$ & $\begin{array}{l}775 \\
850\end{array}$ & $\begin{array}{l}712 \\
733\end{array}$ \\
\hline
\end{tabular}

The regulation of the flexible-bladed machine has been assumed to be such that the flexible device starts to operate at a wind speed of $v=7.5 \mathrm{~m} / \mathrm{s}$.

If it is decided that the machine must be designed to withstand a wind of $50 \mathrm{~m} / \mathrm{s}$., it will be noticed that the normal stresses on the flexible-bladed machine are approximately one-fifth of those on the fixed-bladed machine of the same area; consequentJy the cost of construction is reduced.

Machine with Variable pitch bLades With SERVoMOTOR:

This machine differs from the preceding machine that, in a windmill, the power, and consequently the stresses to which the machine is subjected, vary much more quickly than the speed. The reliability in operation of the machine will be considerably improved by combining the servo-motor system with a flexible system of control of the blades. It will thus be possible, while ensuring a suitable regulation of the power, to support sudden gusts of wind. In fact, a slight variation of pitch of the blades is sufficient, especially at high wind speeds, to modify the stresses considerably; these accidental variations can be supported by the flexible connections, while the average regulation is ensured by the servo-motor and the power governor. 


\section{CONCLUSION}

Of the possible types of windmill, we have retained four which seem to be able to be constructed for large powers.

The fixed-bladed machine is attractive with its simplicity, although the construction of the blades. as a result of the need to use fine profiles, is difficult. Certain reservations must also be made concerning the possibilities of operation at high powers.

The "bicycle wheel" machine, which can be treated in accordance with the principle of either fixed blades or blades of partially variable pitch, has a design based essentially on the use of inexpensive profiled parts. A study of it will show to what extent it is possible to reduce the cost per $\mathrm{kWh}$ by concentrating on the simplification of the processes of manufacture.

The two machines considered with variable pitch blades differ only in their method of control, but the essential part of their design is identical.

The machine with llexible mounting differs from the others by its behaviour in gusts. The machine with the servo-motor meets all the requirements of the generation of electricity on a network; in combination with the flexible mounting it also ensures resistance to gusts and very high winds.

It is, of course, obvious that for all these machines the investigations will have no chance of leading to the desired results, particularly the economic generation of wind power, unless the need for an economic construction is kept constantly in mind and costly mechanical or aerodynamic developments avoided. The cost per $\mathrm{kWh}$ will be brought down to a reasonable level only if the number of installations is large enough to enable the cost of manufacture to be reduced by the adoption of mass production methods.

\section{CONCERNING ISSUE $\mathrm{N}^{\circ} 1,1957$}

We apologise for the unfortunate error which resulted in our failing to make an important acknowledgement in "La Houille Blanche" $n^{\circ} 1,1957$. We should have mentioned that the articles making up this issue were papers presented by theirs authors at the sessions of the Comité Technique de la Société Hydrotechnique de France (S.H.F.) wich took place on November 15th 1956. These sessions were organised in cooperation with the Association Technique pour la production et l'utilisation de l'Energie Nucléaire (A.T.E.N.) with Fluid mechanics and heat transfer as the topic.

We are very happy to have been able to put this valuable contribution at the disposal of our readers and we would like to thank the S.H.F. and the A.T.E.N. on their behalf as well as on our own. 